\title{
A model for the postcollapse equilibrium of cosmological structure: truncated isothermal spheres from top-hat density perturbations
}

\author{
Paul R. Shapiro, ${ }^{1}$ Ilian T. Iliev ${ }^{2}$ and Alejandro C. Raga ${ }^{3}$ \\ ${ }^{1}$ Dept. of Astronomy, The University of Texas at Austin, Austin, TX 78712, USA, E-mail: shapiro@astro.as.utexas.edu \\ ${ }^{2}$ Dept. of Physics, The University of Texas at Austin, Austin, TX 78712, USA, E-mail: iliev@astro.as.utexas.edu \\ ${ }^{3}$ Instituto de Astronomía-Universidad Nacional Autonoma de México, Apdo Postal 70-264, 04510 México D.F., México, \\ E-mail: raga@astroscu.unam.mx
}

20 September 2018

\begin{abstract}
The postcollapse structure of objects which form by gravitational condensation out of the expanding cosmological background universe is a key element in the theory of galaxy formation. Towards this end, we have reconsidered the outcome of the nonlinear growth of a uniform, spherical density perturbation in an unperturbed background universe - the cosmological "top-hat" problem. We adopt the usual assumption that the collapse to infinite density at a finite time predicted by the top-hat solution is interrupted by a rapid virialization caused by the growth of small-scale inhomogeneities in the initial perturbation. We replace the standard description of the postcollapse object as a uniform sphere in virial equilibrium by a more self-consistent one as a truncated, nonsingular, isothermal sphere in virial and hydrostatic equilibrium, including for the first time a proper treatment of the finite-pressure boundary condition on the sphere. The results differ significantly from both the uniform sphere and the singular isothermal sphere approximations for the postcollapse objects. The virial temperature which results is more than twice the previously used "standard value" of the postcollapse uniform sphere approximation but 1.4 times smaller than that of the singular, truncated isothermal sphere approximation. The truncation radius is 0.554 times the radius of the top-hat at maximum expansion, and the ratio of the truncation radius to the core radius is 29.4 , yielding a central density which is 514 times greater than at the surface and $1.8 \times 10^{4}$ times greater than that of the unperturbed background density at the epoch of infinite collapse predicted by the top-hat solution. For the top-hat fractional overdensity $\delta_{L}$ predicted by extrapolating the linear solution into the nonlinear regime, the standard top-hat model assumes that virialization is instantaneous at $\delta_{L}=\delta_{c}=1.686$, i.e. the epoch at which the nonlinear top-hat reaches infinite density. The surface of the collapsing sphere meets that of the postcollapse equilibrium sphere slightly earlier, however, when $\delta_{L}=1.52$. These results will have a significant effect on a wide range of applications of the Press-Schechter and other semi-analytical models to cosmology.
\end{abstract}

We discuss the density profiles obtained here in relation to the density profiles for a range of cosmic structures, from dwarf galaxies to galaxy clusters, indicated by observation and by N-body simulation of cosmological structure formation, including the recent suggestion of a universal density profile for halos in the Cold Dark Matter (CDM) model. The nonsingular isothermal sphere solution presented here predicts the virial temperature and integrated mass distribution of the X-ray clusters formed in the CDM model as found by detailed, 3D, numerical gas and N-body dynamical simulations remarkably well. This solution allows us to derive analytically the numerically-calibrated mass-temperature and radius-temperature scaling laws for

(C) 0000 RAS 
X-ray clusters which were derived empirically by Evrard, Metzler and Navarro from simulation results for the CDM model.

Key words: cosmology: theory - dark matter - galaxies: clusters: general - galaxies: formation - galaxies: haloes - galaxies: kinematics and dynamics

\section{INTRODUCTION}

The theory of structure formation in cosmology must answer the question of how gravitational instability amplified initially small-amplitude density fluctuations in an expanding background universe until the nonlinear structure we see today, from galaxies to clusters of galaxies, emerged. It must explain the number and frequency of these objects, their spatial clustering and relative motions, their internal structure, and the evolution of these properties. The current view is that this structure arose by the complex, hierarchical build-up which resulted from a primordial spectrum of Gaussian-random noise which reached nonlinear amplitude some time after matter began to dominate the total energy density of the universe. The exact nature of this structure formation is not amenable to an analytical solution. As a result, much effort has gone into large-scale numerical simulation by N-body techniques and, more recently, the coupling of N-body and hydrodynamics methods. For many purposes, however, analytical and semi-analytical approximations are possible and serve both as a guide to understanding the complex numerical results and as a crucial means of extrapolating beyond them in order to interpret the predictions of cosmological models in comparison with the observed universe.

The nonlinear evolution of cosmic density fluctuations is often approximated by a model in which the initial linear perturbation is an isolated, uniform sphere outside of which the matter is unperturbed - the "top-hat" perturbation - for which an exact solution is possible (e.g. Peebles 1980, Peebles 1993, Padmanabhan 1993; and references therein). For many purposes, a critical part of the application of this top-hat model involves the assumption that the collapse to infinite density predicted by the solution is interrupted by a rapid relaxation to virial equilibrium at a finite density, as a result of the growth of initially small inhomogeneities in the density distribution. For example, many applications of the Press-Schechter approximation (Press and Schechter 1974) for the rate of formation of objects of a given mass per unit time due to the growth of Gaussian-random-noise density perturbations involve this assumption of postcollapse virialization (see, for example, Monaco 1997 for a review and references).

We have reconsidered the postcollapse virial equilibrium of the top-hat model. We improve upon the standard assumption of a uniform sphere for the virialized final state by a self-consistent model involving a nonsingular, truncated isothermal sphere ("TIS") in hydrostatic and virial equilibrium which takes proper account of the finite-pressure boundary condition on the sphere. The results differ significantly from those of the standard uniform sphere ("SUS") approximation for the final virialized object. They also differ significantly from those of the approximation of a singular isothermal sphere ("SIS") for the final object.

An outline of the paper is as follows. In $\S 2$ we describe the top-hat model and the standard approach to the final virialized object in the SUS approximation. In $\S$ we describe the hydrostatic equilibrium solutions for isothermal spheres. An application of the virial theorem to characterize the equilibrium of a truncated isothermal sphere, including a proper account of the finite boundary pressure, is described in $\S$. A minimum-energy argument which selects a unique TIS solution for a given boundary pressure is presented in $\S$. This solution is used to specify the postcollapse TIS which results from any given top-hat density perturbation in $\S 6$. An independent calculation of this postcollapse TIS solution is presented in $\S$ 7. based on the self-similar infall solution of Bertschinger (1985), with results which agree closely with the minimum-energy argument, thereby confirming it. Our results are summarized and discussed in $\S$. Our summary in $\S .1$ includes a complete prescription, with scaling laws and an approximate fitting formula, for using our minimum-energy TIS solution in combination with the top-hat perturbation solution to describe the cosmological formation and postcollapse equilibrium structure of any object as a function of its mass and collapse epoch. In $\S 8$, we compare the predictions of this TIS solution with those of the SUS and SIS approximations. A comparison of the TIS density profile with existing results of numerical simulations of cosmic structure formation and with observations for a range of cosmic structures from dwarf galaxies to galaxy clusters is made in $\S$ 8.3. This includes a discussion of the minimum-energy TIS solution in relation to the suggestion by Navarro, Frenk, and White $(1996,1997)$ of a universal form for the density profiles of haloes of collisionless dark matter formed in a hierarchical clustering model like the Cold Dark Matter (CDM) model, based upon the results of numerical N-body simulation. Finally, in $\S 8.4$, we compare our predicted virial temperature with the results of numerical gas dynamical simulations of X-ray cluster formation in the CDM model, and derive analytically the mass-radius-temperature scaling laws for X-ray clusters determined empirically by Evrard, Metzler, and Navarro (1996) from gas dynamical and N-body simulations of the CDM model. 


\section{THE STANDARD APPROACH TO THE TOP-HAT MODEL}

All the results in this section are well known, but due to their importance in what follows we shall summarize them here.

\subsection{Before collapse: the exact nonlinear solution}

The spherical top-hat model, a spherical perturbation of uniform overdensity (Gunn \& Gott 1972), affords considerable insight into the dynamics of gravitational growth of cosmic structure, while still having an exact, analytical or semi-analytical, solution in certain cosmological models (cf. Peebles 1980). In what follows we shall consider top-hat perturbations only in the Einsteinde Sitter model.

Based on its high degree of symmetry, the collapse of a top-hat density perturbation can be described in detail solely in terms of its overdensity with respect to the background. From the Birkhoff theorem, the equation describing this evolution is identical to the Friedmann equation for a universe with the corresponding mean density. The linearized version of this equation describes the linear evolution of the density perturbation $\delta_{L}(t)$ and has a growing solution $\delta_{L}=\delta_{i}\left(t / t_{i}\right)^{2 / 3}$. Using this solution we can write the equation for the density contrast in terms of $\delta_{L}(t)$, whose solution is independent of the initial density contrast $\delta_{i}$ and the corresponding time $t_{i}$ and hence describes the general, scale-invariant collapse solution. In parametric form the solution reads (e.g. Padmanabhan 1993)

$$
\delta=\frac{9}{2} \frac{(\theta-\sin \theta)^{2}}{(1-\cos \theta)^{3}}-1, \quad \delta_{L}=\frac{3}{5}\left(\frac{3}{4}\right)^{2 / 3}(\theta-\sin \theta)^{2 / 3} .
$$

The critical density contrast $\delta_{c}$ is defined as the linear solution extrapolated to the epoch at which the nonlinear solution predicts an infinite density. As described above, this effectively is the time of collapse in scale-free units. The values obtained at turnaround ( $\theta=\pi$, indicated by subscript " $m$ " to denote "maximum expansion") and at collapse time $(\theta=2 \pi$, denoted by subscript $c$ ) are:

$$
\delta_{L m}=\frac{3}{5}\left(\frac{3 \pi}{4}\right)^{2 / 3} \approx 1.0624, \quad \delta_{m}=\frac{9 \pi^{2}}{16}-1 \approx 4.5517, \quad \delta_{c}=\frac{3}{5}\left(\frac{3 \pi}{2}\right)^{2 / 3} \approx 1.6865, \quad \delta\left(\delta_{c}\right)=\infty .
$$

Henceforth, we shall refer to $z_{\text {coll }}$ as the redshift which corresponds to the epoch of infinite collapse, at which $\delta=\infty$, at time $t_{\text {coll }}$.

\subsection{After collapse: uniform sphere in virial equilibrium}

As shown above, a perfectly symmetric top-hat collapse results in a singularity at $\delta_{L}=\delta_{c}$, which does not lead to the formation of virialized structures. To overcome this problem, it is usually assumed that the actual collapse is slightly inhomogeneous, and therefore the top-hat does not collapse to infinite density, but instead, by means of processes like violent relaxation, relaxes to form a static, virialized structure. Assuming that the total energy is conserved during the collapse, we can connect the initial top-hat to the final state as follows.

The standard approach is to assume that the collapse of the top-hat to infinite density is interrupted by a rapid equilibration at $\delta_{L}=\delta_{c}$ which results in another uniform sphere in virial equilibrium. We solve for the final radius $r_{\mathrm{vir}}$ of the virialized sphere by applying the virial theorem to the final state and expressing the conserved total energy $E$ of the sphere in terms of the radius $r_{m}$ at maximum expansion. At the point of maximum expansion (which always exists for $\Omega \geq 1$ ) the sphere is cold and at rest, so its energy is entirely gravitational potential energy which, for uniform sphere of mass $M_{0}$ and radius $r_{m}$ is just

$E=W_{m}=-\frac{3}{5} \frac{G M_{0}^{2}}{r_{m}}$

After the system settles down to a virial equilibrium its potential energy is

$W_{\mathrm{vir}}=-\frac{3}{5} \frac{G M_{0}^{2}}{r_{\mathrm{vir}}}$

According to the virial theorem

$0=2 K+W$

where $K=U_{\mathrm{th}}+T_{\text {kin }}, U_{\mathrm{th}}$ and $T_{\text {kin }}$ are the thermal and kinetic energy, respectively. Together with the conservation of energy, $E=K+W$, this implies $E=-W / 2$, and, therefore, the total energy is related to the virial radius according to

$E=-\frac{3}{10} \frac{G M_{0}^{2}}{r_{\text {vir }}}$

Equating the total energy $E$ in equations (3) and (6) yields

(C) 0000 RAS, MNRAS 000, 000-000 
$r_{\mathrm{vir}}=\frac{r_{m}}{2}$

This is the well-known result for the size of the virialized object in the SUS approximation. The virial temperature and velocity are derived as follows. The kinetic energy in the virialized state is

$K_{\text {vir }}=E-W_{\text {vir }}=\frac{3}{10} \frac{G M_{0}^{2}}{r_{\text {vir }}}$.

This kinetic energy is the energy of internal motions only $\left(T_{k i n}=0\right)$. To obtain the equivalent temperature we express the thermal energy

$U_{\mathrm{th}}=\frac{3}{2} \frac{k_{B} T}{m} M_{0}$

where $m$ is the mean mass per gas particle. If $m_{H}$ is the mass of a hydrogen atom, then $m=\mu m_{H}$ where $\mu$ is the mean molecular weight. Therefore the equivalent virial temperature is

$T=\frac{1}{5} \frac{G M_{0} m}{k_{B} r_{\mathrm{vir}}}=\frac{2}{5} \frac{G M_{0} m}{k_{B} r_{m}}$.

Henceforth, we shall refer to the virial temperature in equation (10) as $T_{S U S}$. For the case of a collisionless gas, we replace the virial temperature above by the virial velocity dispersion,

$\sigma^{2}=\frac{\left\langle v^{2}\right\rangle}{3}=\frac{k_{B} T}{m}$

According to this approach, the properties of the final virialized uniform sphere are related to those of the top-hat perturbation which created it as follows, as summarized by Padmanabhan(1993), except we use $h=H_{0} /\left(100 \mathrm{~km} \mathrm{~s}^{-1} \mathrm{Mpc}^{-1}\right)$ rather than $h_{0.5}=2 h$, and we include the explicit dependence on the mean molecular weight $\mu$, where $\mu=0.59(1.22)$ for an ionized (neutral) gas of $\mathrm{H}$ and He with $[\mathrm{He}] /[\mathrm{H}]=0.08$ by number:

$r_{m}=338.3\left(\frac{M}{10^{12} M_{\odot}}\right)^{1 / 3}\left(1+z_{\text {coll }}\right)^{-1} h^{-2 / 3} \mathrm{kpc}$,

$\left(r_{\text {vir }}\right)_{S U S}=169.2\left(\frac{M}{10^{12} M_{\odot}}\right)^{1 / 3}\left(1+z_{\text {coll }}\right)^{-1} h^{-2 / 3} \mathrm{kpc}$

$T_{S U S}=6.160 \times 10^{5} \mu\left(\frac{M}{10^{12} M_{\odot}}\right)^{2 / 3}\left(1+z_{\mathrm{coll}}\right) h^{2 / 3} \mathrm{~K}$

$\left(v_{c}\right)_{S U S}=5^{1 / 2} \sigma=\left(\frac{5}{3}\right)^{1 / 2}\left\langle v^{2}\right\rangle^{1 / 2}=159.4\left(\frac{M}{10^{12} M_{\odot}}\right)^{1 / 3}\left(1+z_{\mathrm{coll}}\right)^{1 / 2} h^{1 / 3} \mathrm{~km} \mathrm{~s}^{-1}$,

$(\bar{\rho})_{S U S}=18 \pi^{2}\left(1+z_{\mathrm{coll}}\right)^{3} \rho_{b 0}$

where $v_{c}=\left(G M / r_{\text {vir }}\right)^{1 / 2}$ is the circular velocity at $r_{\text {vir }}$.

This approach is commonly used to calculate the virial temperature and radius. However it is not a realistic model, since it assumes isothermality, uniform density, and the absence of external pressure, which are clearly incompatible. A more realistic approach is to assume a final state of hydrostatic equilibrium (or the corresponding state with an isotropic Maxwellian velocity distribution in the case of collisionless particles), which we will do in the next section.

\section{ISOTHERMAL SPHERES}

The final virialized object is decoupled from the expanding cosmological background from which it condensed. Hence, when we describe it as an isothermal sphere in hydrostatic equilibrium, we do so in the usual non-cosmological way (cf. Binney and Tremaine 1987). The hydrostatic equilibrium equation, $\nabla p=\rho \mathbf{g}$, in the case of spherical symmetry becomes

$T \frac{k_{B}}{m} \frac{d \rho}{d r}=-\rho g=-\rho \frac{G M(r)}{r^{2}}$

where $M(r)$ is the mass inside radius $r$. Multiplying equation (17) by $r^{2} m / \rho k_{B} T$, and taking the derivative with respect to $r$, we obtain

$\frac{d}{d r}\left(r^{2} \frac{d(\ln \rho)}{d r}\right)=-4 \pi \frac{G m}{k_{B} T} r^{2} \rho$

Let us consider the case of collisionless particles, too. The Poisson equation for the gravitational potential in the case of spherical symmetry is

(C) 0000 RAS, MNRAS 000, 000-000 
$\frac{1}{r^{2}} \frac{d}{d r}\left(r^{2} \frac{d \Psi}{d r}\right)=-4 \pi G \rho$.

The equilibrium velocity distribution of the particles is a Maxwellian distribution given by distribution is

$f(v)=\frac{\rho_{0}}{\left(2 \pi \sigma^{2}\right)^{3 / 2}} \exp \left(\frac{\Psi-v^{2} / 2}{\sigma^{2}}\right)$,

where $\rho_{0}$ is the central density if we take $\Psi(r=0)=0$, and $\sigma$ is the one dimensional velocity dispersion. After integrating over velocity we obtain

$\rho=\int f(v) d \mathbf{v}=\rho_{0} e^{\Psi / \sigma^{2}}$.

Therefore

$\Psi=\sigma^{2} \ln \left(\frac{\rho}{\rho_{0}}\right)$

which we substitute in equation (19), obtaining

$\frac{d}{d r}\left(r^{2} \frac{d(\ln \rho)}{d r}\right)=-\frac{4 \pi}{\sigma^{2}} G \rho r^{2}$.

By calculating the mean squared velocity we obtain

$\left\langle v^{2}\right\rangle=3 \sigma^{2}$.

The equivalent temperature can be calculated from

$\frac{\left\langle v^{2}\right\rangle}{2}=\frac{3}{2} \frac{k_{B} T}{m}$

obtaining for $\sigma$ :

$\sigma^{2}=\frac{k_{B} T}{m}$.

A comparison of equation (23) with equation (18) using equation (26) shows they are identical. Hence, the structure of an isothermal self-gravitating fluid sphere is identical with the structure of a collisionless 3D spherical system in equilibrium.

To make equation (23) nondimensional, we introduce new variables

$\tilde{\rho}=\frac{\rho}{\rho_{0}}, \quad \zeta=\frac{r}{r_{0}}$,

where $\rho_{0}$ is the central density, and we choose

$r_{0}=\sigma /\left(4 \pi G \rho_{0}\right)^{1 / 2}$.

In these variables equation (23) becomes

$\frac{d}{d \zeta}\left(\zeta^{2} \frac{d(\ln \tilde{\rho})}{d \zeta}\right)=-\tilde{\rho} \zeta^{2}$.

We must solve equation (29) with boundary conditions

$\tilde{\rho}(0)=1, \quad \frac{d \tilde{\rho}}{d \zeta}(0)=0$.

A simple expression which satisfies equation (29) exactly, but violates the boundary conditions in equations (30), often used in models due to its simplicity, is that of a singular isothermal sphere, for which

$\rho(r)=\frac{\sigma^{2}}{2 \pi G r^{2}}$,

and

$\sigma^{2}=\frac{1}{2} \frac{G M(r)}{r}=$ constant.

However, any solution of both equations (29) and (30) must have a core (i.e. $r_{0} \neq 0$ ), and, while equations (31) and (32) correspond to the asymptotic limit of the solutions of equations (29) and (30) as $r_{0} \rightarrow 0$, they are not good approximations in general. We will compare our results for the nonsingular case with that of the singular isothermal sphere in what follows.

The isothermal sphere has infinite mass $(M \propto r$ for large radii). Hence, in order to describe some realistic finite structure in terms of this model, we must truncate the sphere at some radius, $r_{t}$. The total mass $M_{0}$ of the isothermal sphere is then 
$M_{0}=M\left(r_{t}\right)=\int_{0}^{r_{t}} 4 \pi \rho(r) r^{2} d r=4 \pi \rho_{0} r_{0}^{3} \tilde{M}\left(\zeta_{t}\right)$

where $\zeta_{t}=r_{t} / r_{0}$ and $\tilde{M}$ is the dimensionless mass:

$\tilde{M}\left(\zeta_{t}\right) \equiv \frac{M\left(r_{t}\right)}{4 \pi r_{0}^{3} \rho_{0}}=\int_{0}^{\zeta_{t}} \tilde{\rho}(\zeta) \zeta^{2} d \zeta$.

Using equation (28), we obtain

$M\left(r_{t}\right)=\frac{\sigma^{3}}{G\left(4 \pi G \rho_{0}\right)^{1 / 2}} \tilde{M}_{t}$,

where $\tilde{M}_{t} \equiv \tilde{M}\left(\zeta_{t}\right)$. Solving for $\sigma^{2}$, we obtain

$\sigma^{2}=G\left(4 \pi \rho_{0}\right)^{1 / 3}\left(\frac{M_{0}}{\tilde{M}_{t}}\right)^{2 / 3}$,

while equation $(26)$ yields the gas temperature:

$T=\frac{G m}{k_{B}}\left(4 \pi \rho_{0}\right)^{1 / 3}\left(\frac{M_{0}}{\tilde{M}_{t}}\right)^{2 / 3}$.

The existence of a truncation radius leads to the necessity of an external pressure to keep the system in equilibrium. This requires a significant correction to the form of the virial theorem stated in the previous section and, accordingly, to all of the other results obtained there.

\section{THE VIRIAL THEOREM FOR TRUNCATED ISOTHERMAL SPHERES AND ITS CONSEQUENCES}

Let us consider the general isothermal sphere density profile $\rho(r)$, obtained in the previous section. From the ideal gas law, the pressure inside as a function of the radius is

$p(r)=\frac{k_{B} T}{m} \rho(r)=\sigma^{2} \rho(r)$,

and at the outer edge

$p_{t}=p\left(r_{t}\right)=\sigma^{2} \rho\left(r_{t}\right)$.

The mean pressure inside the sphere is

$\bar{p}=\frac{\int p d V}{\int d V}=\frac{3 \int_{0}^{\zeta_{t}} \tilde{\rho}(\zeta) \zeta^{2} d \zeta}{\zeta_{t}^{3} \tilde{\rho}\left(\zeta_{t}\right)} p_{t}=\frac{3 \tilde{M}\left(\zeta_{t}\right)}{\zeta_{t}^{3} \tilde{\rho}\left(\zeta_{t}\right)} p_{t} \equiv \alpha\left(\zeta_{t}\right) p_{t}$

where $\zeta_{t} \equiv r_{t} / r_{0}$. A plot of $\alpha\left(\zeta_{t}\right)$ is shown on Figure 1 . The limit $\zeta_{t}=\infty, \alpha=3$ is the case of a singular isothermal sphere, which will be considered in more detail below.

The virial theorem for a static sphere in the presence of a surface pressure reads

$0=2 K+W+S_{p}$,

where $W$ is the gravitational potential energy, $\mathrm{K}$ is just $U_{t h}$, the thermal energy, and $S_{p}$ is the surface pressure term. The thermal energy for a gas with ratio of specific heats $\gamma=5 / 3$ is given by

$U_{t h}=\frac{3}{2} \int p d V=\frac{3}{2} \alpha\left(\zeta_{t}\right) p_{t} V$,

where $V$ is the total volume. The surface term is equal to

$S_{p}=-\int p \mathbf{r} \cdot d \mathbf{S}=-3 V p_{t}$.

Hence the potential energy is

$W=-2 U_{t h}-S_{p}=-2 \frac{\alpha-1}{\alpha} U_{t h}$

and the total energy is

$E=\frac{2-\alpha}{\alpha} U_{t h}$. 


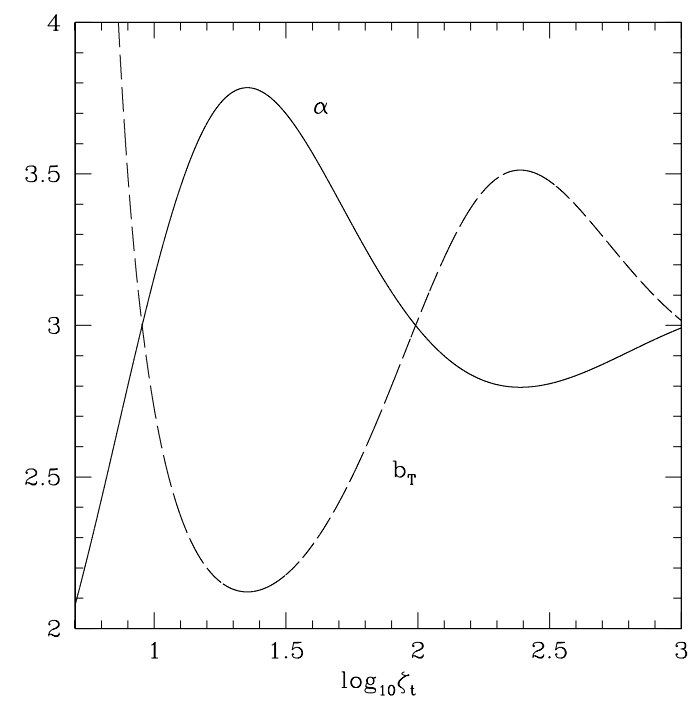

Figure 1. The ratio of the mean pressure to the boundary pressure $\alpha=\bar{p} / p_{t}$ for an isothermal sphere truncated at radius $r_{t}$ (solid curve), is plotted against the dimensionless truncation radius $\zeta_{t}=r_{t} / r_{0}$. The ratio of the temperature of the isothermal sphere to that of a uniform sphere with the same mass and total energy according to the SUS approximation, $b_{T}=T / T_{S U S}$, is plotted as well (dashed curve).

In order to satisfy the virial theorem, the condition that $U_{t h}>0$ (and $E<0$ ) requires $\alpha>2$, while Figure 1 indicates that $\alpha<3.7846$ in general. The virial temperature of the isothermal sphere is

$T=\frac{2 \alpha}{5(\alpha-2)} \frac{G M_{0} m}{k_{B} r_{m}}$

which we shall henceforth refer to as $T_{T I S}$ 风. Since $\alpha /(\alpha-2)>1$ for any $\alpha$, the temperature $T_{T I S}$ is always higher than $T_{S U S}$, the standard value for a uniform sphere shown in equation (10). If we write $T_{T I S}=b_{T} T_{S U S}$, we find $b_{T}>2.13$. This temperature correction factor is also plotted in Figure 1 .

The virial radius $r_{\text {vir }}$ in this case is just the size of the truncated isothermal sphere, or $r_{\text {vir }}=r_{t}$. For comparisons with the results of dynamical calculations of the formation of such an equilibrium object, we should interpret $r_{\mathrm{vir}}\left(\right.$ and $\left.r_{t}\right)$ as the radius inside which hydrostatic equilibrium holds (Cole \& Lacey 1996). Care should be taken not to confuse this definition of $r_{\text {vir }}$ with the one that is most often used previously when identifying collapsed haloes in simulations, that of the radius inside which the mean overdensity is $18 \pi^{2} \approx 178$, as predicted by the top-hat solution if $r_{\mathrm{vir}}=r_{m} / 2$. We will demonstrate below that our solution for $r_{\mathrm{vir}}=r_{t}$ is not far from this value, even though the structure of the TIS is very different from that of the SUS approximation. This is consistent with results obtained using numerical simulations (Cole \& Lacey 1996).

For some purposes, the approximation of a truncated, singular isothermal sphere ("SIS") has been used, instead of the exact solution, since it is algebraically simpler and the results can be obtained analytically, even though this approximation cannot be correct at the origin. For comparison with the nonsingular TIS results and as further illustration of the importance of the correction for finite boundary pressure discussed above, we calculate the virial temperature for such truncated, but singular isothermal spheres as follows. Using equation (31) and the ideal gas law, the pressure at radius $r$ is given by

$p(r)=p_{t}\left(\frac{r}{r_{t}}\right)^{-2}$

where the boundary pressure at $r=r_{t}$ is given by

$p_{t}=\frac{k_{B} T}{m} \rho_{t}=\sigma^{2} \rho_{t}=\frac{\sigma^{4}}{2 \pi G r_{t}^{2}}$.

The average pressure inside the truncated isothermal sphere is then just

$\bar{p}=3 p_{t}$,

(i.e. $\alpha=3$ ). The thermal energy in this case is given by

* Henceforth. the notation "TIS" shall refer to a solution of the isothermal Lane-Emden equation with nonsingular boundary conditions of equation (30) at the origin 
$U_{t h}=\frac{3}{2} \int p d V=\frac{9}{2} p_{t} V=\frac{3 \sigma^{4} r_{t}}{G}=\frac{3}{4} \frac{G M_{0}^{2}}{r_{t}}$,

while the surface pressure term in the virial theorem equation is given by

$S_{p}=-\int p \mathbf{r} \cdot d \mathbf{S}=-4 \pi r_{t}^{3} p_{t}=-\frac{2 \sigma^{4} r_{t}}{G}=-\frac{1}{2} \frac{G M_{0}^{2}}{r_{t}}$.

According to the virial theorem, the potential energy is, therefore, given by

$W=-2 U_{t h}-S_{p}=-\frac{4 \sigma^{4} r_{t}}{G}=-\frac{4}{3} U_{t h}=-\frac{G M_{0}^{2}}{r_{t}}$,

and the total energy becomes

$E=U_{t h}+W=-\frac{U_{t h}}{3}=-\frac{1}{4} \frac{G M_{0}^{2}}{r_{t}}$.

The SIS virial temperature is calculated as above for the nonsingular case according to equation (46), except that $\alpha=3$ for the singular case, which yields

$T=T_{S I S}=\frac{6}{5} \frac{G M_{0} m}{k_{B} r_{m}}$,

This virial temperature is a factor of $b_{T}=\alpha /(\alpha-2)=3$ times larger than $T_{S U S}$ in equation (10) given by the SUS approximation. Equations (50) and (54) then yield the truncation radius for this SUS case, $r_{t}=(5 / 12) r_{m}$. Hence, in the limiting case of a singular, truncated isothermal sphere, the correction to the virial temperature resulting from the finite boundary pressure term is of considerable importance, while the difference between the actual radius of the isothermal sphere and the virial radius of the SUS approximation (i.e. $r_{v i r, S U S}=r_{m} / 2$ ) is more modest. In what follows, we shall derive the corrections for the actual, nonsingular truncated isothermal sphere which results from the top-hat collapse.

\section{CHOOSING A UNIQUE PROFILE: THE MINIMUM-ENERGY SOLUTION}

\subsection{The solution}

As shown above, the truncated isothermal sphere solutions form a one-parameter family, described by $\zeta_{t}=r_{t} / r_{0}-$ the truncation radius in units of the core radius. Specifying $\zeta_{t}$, the total mass, and total energy completely determines the solution. Alternatively, we can specify the mass, the total energy and the applied external pressure $p_{t}$. Using these parameters, we can implicitly express the truncation radius as follows. The dimensionless density at $\zeta_{t}$ is

$\tilde{\rho}\left(\zeta_{t}\right)=\frac{\rho_{t}}{\rho_{0}}=\frac{p_{t}}{\sigma^{2} \rho_{0}}=\frac{m p_{t}}{k_{B} T \rho_{0}}$.

From equations (28) and (37) we obtain

$$
\begin{aligned}
r_{0} & =\frac{M_{0}}{\tilde{M}_{t}} \frac{m G}{k_{B} T}, \\
\rho_{0} & =\frac{1}{4 \pi}\left(\frac{\tilde{M}_{t}}{M_{0}}\right)^{2}\left(\frac{k_{B} T}{m G}\right)^{3},
\end{aligned}
$$

and, by substituting this $\rho_{0}$ in equation (55), we obtain

$\tilde{\rho}\left(\zeta_{t}\right)=4 \pi\left(\frac{m}{k_{B} T}\right)^{4} p_{t}\left(\frac{M_{0}}{\tilde{M}_{t}}\right)^{2} G^{3}$.

Using equation (45), the total energy can be written as

$E=\frac{2-\alpha}{\alpha} \frac{3 k_{B} T}{2 m} M_{0}$

and, together with equation (57), we finally obtain

$E=\frac{3}{2}\left(4 \pi G^{3} p_{t} M_{0}^{6}\right)^{1 / 4} \tilde{E}\left(\zeta_{t}\right)$,

where

$\tilde{E}\left(\zeta_{t}\right)=\frac{2-\alpha\left(\zeta_{t}\right)}{\alpha\left(\zeta_{t}\right)\left[\tilde{\rho}\left(\zeta_{t}\right) \tilde{M}^{2}\left(\zeta_{t}\right)\right]^{1 / 4}}$.

Similarly, we can define a dimensionless external pressure $\tilde{p}$ : 


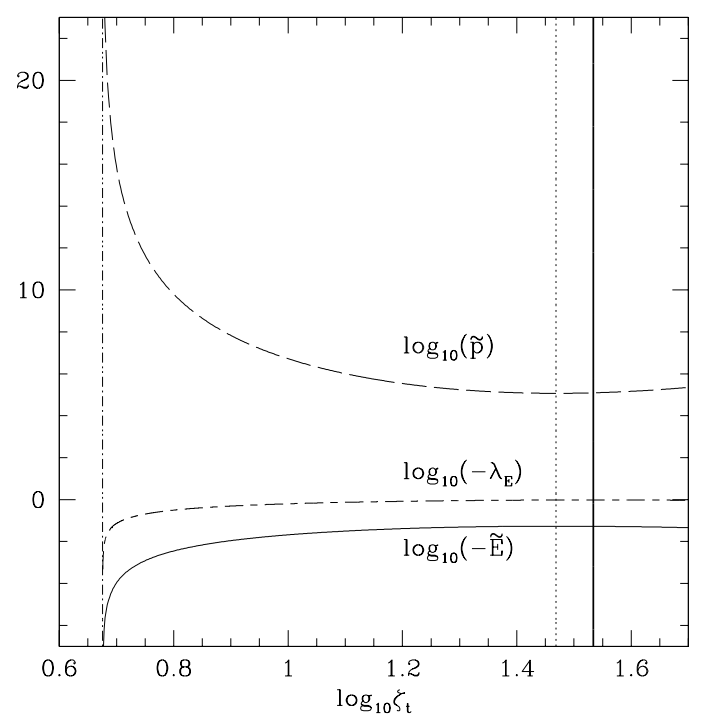

Figure 2. Logarithms of the dimensionless energy $\tilde{E}\left(\zeta_{t}\right)$ (solid curve), external pressure $\tilde{p}\left(\zeta_{t}\right)$ (long-dashed curve), and radius $\left|\lambda_{E}\left(\zeta_{t}\right)\right|$ (long-dashed - short-dashed curve) versus dimensionless truncation radius $\zeta_{t}$ for a truncated isothermal sphere. The vertical dot-dashed line indicates the value $\zeta_{t}=4.738$ below which $E>0$ and a TIS cannot exist. The vertical dotted line indicates the value $\zeta_{t}=29.4$ at which the energy is minimal. The vertical thick line indicates the boundary of stability for the TIS solution $\left(\zeta_{t}=34.2\right.$, see $\S 5.2$. TIS solutions are unstable to the right of this line.

$\tilde{p}\left(\zeta_{t}\right) \equiv\left[\tilde{E}\left(\zeta_{t}\right)\right]^{-4}$

where

$p_{t}=\frac{(2 E / 3)^{4}}{4 \pi G^{3} M_{0}^{6}} \tilde{p}\left(\zeta_{t}\right)$

We have plotted the dependence of the dimensionless total energy $\tilde{E}$ and the dimensionless external pressure $\tilde{p}$ on $\zeta_{t}$ in Figure 2. In order to indicate the dependence of the size of the sphere on $\zeta_{t}$, we nondimensionalize the radius $r_{t}$, according to

$-\lambda_{E} \equiv-\frac{r_{t} E}{G M_{0}^{2}}=\frac{3}{2} \frac{\alpha-2}{\alpha} \frac{\zeta_{t}}{\tilde{M}_{t}}$

and plot $-\lambda_{E}$ against $\zeta_{t}$ in Figure 8 , as well. This definition of $\lambda_{E}$ also corresponds to the familiar dimensionless energy parameter used in discussions of the stability of isothermal spheres (see $\S 5.2$ below).

For a given mass $M_{0}$ and external pressure $p_{t}$, the solution is specified uniquely only if we can uniquely identify a special value of $\zeta_{t}$, or equivalently, of $E$. Apparently, for any truncated isothermal sphere of mass $M_{0}$ which is confined by a given external pressure $p_{t}$, there is a unique value of $\zeta_{t}$ which minimizes the total energy $E$. We shall make the reasonable anzatz that this minimum-energy solution is the unique TIS solution preferred in nature as the outcome of the virialization of the sphere in the presence of a fixed external pressure. We offer evidence to support this anzatz in $\S$ for the cosmological top-hat problem. It is possible that this is a general result for any TIS formed by relaxation in the presence of a fixed external pressure, but we are only concerned here with spheres that evolve from cosmological initial conditions.

The minimum value of $E$ as a function of $\zeta_{t}$ for a given $p_{t}$ is found by minimizing the dimensionless energy $\tilde{E}\left(\zeta_{t}\right)$ in equation (60). This occurs for $\zeta_{t}=29.4$, for which $\tilde{E}\left(\zeta_{t}\right)=-0.2816, \alpha\left(\zeta_{t}\right)=3.73, \tilde{M}_{t}=61.485$, and $\tilde{\rho}\left(\zeta_{t}\right)=1.946 \times 10^{-3}$. For a given mass $M_{0}$ and boundary pressure $p_{t}$, the full details of the TIS solution are described in terms of these quantities as follows. The total energy $E$ is calculated from equation (59), resulting in

$E=\frac{3}{2}\left(\frac{2-\alpha}{\alpha}\right)\left(\frac{4 \pi}{\tilde{\rho}_{t} \tilde{M}_{t}^{2}}\right)^{1 / 4} G^{3 / 4} p_{t}^{1 / 4} M_{0}^{3 / 2}=-0.795 G^{3 / 4} p_{t}^{1 / 4} M_{0}^{3 / 2}$.

The temperature $T$ is obtained from equation $(58)$ and (59), according to

$T=\left(\frac{4 \pi}{\tilde{\rho}_{t} \tilde{M}_{t}^{2}}\right)^{1 / 4} \frac{m}{k_{B}} G^{3 / 4} p_{t}^{1 / 4} M_{0}^{1 / 2}=14.4 \frac{m}{k_{B}} G^{3 / 4} p_{t}^{1 / 4} M_{0}^{1 / 2}$.

Finally, the core radius $r_{0}$ and the central density $\rho_{0}$ are calculated from equations $(56)$ and $(65)$ :

$r_{0}=\left(\frac{\tilde{\rho}_{t}}{4 \pi \tilde{M}_{t}^{2}}\right)^{1 / 4} G^{1 / 4} p_{t}^{-1 / 4} M_{0}^{1 / 2}=1.42 \times 10^{-2} G^{1 / 4} p_{t}^{-1 / 4} M_{0}^{1 / 2}$, 
and

$\rho_{0}=\left(\frac{\tilde{M}_{t}^{2}}{4 \pi \tilde{\rho}_{t}^{3}}\right)^{1 / 4} G^{-3 / 4} p_{t}^{3 / 4} M_{0}^{-1 / 2}=449.5 G^{-3 / 4} p_{t}^{3 / 4} M_{0}^{-1 / 2}$.

The size of the TIS is then just

$r_{t}=\zeta_{t} r_{0}=29.4 r_{0}$

with $r_{0}$ given by equation (56). The dimensionless radius for this value of $\zeta_{t}$ corresponds then to $\lambda_{E}=-0.3326$.

Apparently, the core of the truncated isothermal sphere is very small compared to its overall size. Nevertheless, the profile is quite different from that of a singular isothermal sphere, in that it has a higher average pressure, lower temperature and a core.

\subsection{Stability}

The problem of the stability of truncated isothermal spheres has been discussed by several authors (e.g. Antonov 1962, Lynden-Bell and Wood 1968, Katz 1978, Binney and Tremaine 1987, Padmanabhan 1989, 1990). According to this literature, our unique minimum energy solution above is stable, as follows. The case at hand is that of a sphere bounded by a thermally insulating wall, distinct from the case of a sphere in contact with a heat bath. The family of allowed TIS solutions of the Lane-Emden equation can be parameterized by one variable, $\zeta_{t}$ or, equivalently, by the combination of parameters given by the ratio of densities measured at $r_{0}$ and $r_{t}, \mathcal{R}=\rho_{0} / \rho_{t}$ and the dimensionless energy $\lambda_{E} \equiv r_{t} E / G M^{2}$. For gravitationally bound spheres $(E<0)$, there are no equilibrium solutions for $\lambda_{E}<-0.335$. Only for $\lambda_{E}>-0.335$ are isothermal spheres possible. For large enough density contrast between the center and edge of the sphere, however, equilibria exist but are unstable. In particular, as first discovered by Antonov (1962) and later discussed in detail in Lynden-Bell and Wood (1968) - who called this "the gravothermal catastrophe" - isothermal spheres with $\lambda_{E}>-0.335$ and $\mathcal{R}>708.61$ (corresponding to $\zeta_{t}=34.2$ ) can exist as solutions of the Lane-Emden equation but are unstable. Such spheres are metastable configurations, for which entropy extrema exist but are not local maxima. Only in the intermediate regime, where $\lambda_{E}>-0.335$ and $\mathcal{R}<708.61$, can isothermal spheres both exist and be stable. In this regime, the TIS can be a state of local maximum of the entropy. We note that our unique, minimum-energy solution for the TIS in the presence of a fixed boundary pressure $p_{t}$, described above, satisfies the stability requirement for avoiding the gravothermal catastrophe, since $\zeta_{t}=29.40, \mathcal{R}=\left[\tilde{\rho}\left(\zeta_{t}\right)\right]^{-1}=514$, and $\lambda_{E}=-0.3326$.

Finally, we note that in the TIS solution derived here, the gas temperature is spatially uniform but the equation of state of this gas is not an isothermal one (i.e. changes of density for a given fluid element do not leave the temperature unchanged). In the latter case, the stability is analyzed by considering isothermal spheres in contact with a heat bath, rather than the thermally insulated spheres described above. For a gas with an isothermal equation of state, isothermal spheres are found to be unstable for density contrasts below that of the gravothermal catastrophe limit. This analysis does not apply to our minimum-energy solution above, however. Hence, we do not need to consider the classical results of Ebert (1955, 1957), Bonnor (1956) and McCrea (1957) which deal with the problem of gas spheres bounded by a given surface pressure in thermal equilibrium with an external heat bath, which differs from the present case.

\section{THE TRUNCATED ISOTHERMAL SPHERE FORMED BY TOP-HAT COLLAPSE}

The unique TIS solution found in $\S 5$ by minimizing the total energy of the sphere for a given boundary pressure $p_{t}$ can be applied to the top-hat problem, as follows. The virialized object which results from the collapse of a given top-hat density perturbation must have the mass of the top-hat and the same total energy as the top-hat before it collapsed and virialized. Fixing the mass $M_{0}$ and the energy $E$ of the TIS which results from the collapse of the top-hat amounts to fixing the boundary pressure $p_{t}$ as that value which makes the TIS with this total energy $E$ correspond to the minimum-energy solution described above. Hence, the TIS which results from top-hat collapse must have $\zeta_{t}=29.4$ and $\alpha=3.73$, as found in $\S$. This means that the virial temperature $T_{T I S}$ is larger than that found by the SUS approach, $T_{S U S}$, by the factor $b_{T}=\alpha /(\alpha-2)=2.16$. A plot of $\rho / \rho_{S U S}$ as a function of $r / r_{t}$, demonstrating the significant difference between the two density profiles is shown on Figure 3 .

This conservation of the energy $E$ of the top-hat before and after its collapse and virialization assumes that there is no extra $p d V$-work that needs to be taken into account due to the presence of the external boundary pressure $p_{t}$ which would otherwise alter the final total energy $E$ compared with its initial value before collapse. This is appropriate for the case at hand, since the collapse prior to the epoch of virialization is that of a cold, pressure-free gas which collapses supersonically. As in the well-known, self-similar, spherical infall solution of Bertschinger (1985), the original energy is converted from potential energy at maximum expansion to a mixture of infall kinetic energy and potential energy during infall, with a negligible share of the energy going into compressional heating. In that solution, the infall is halted by a strong shock. At this shock, the kinetic energy 


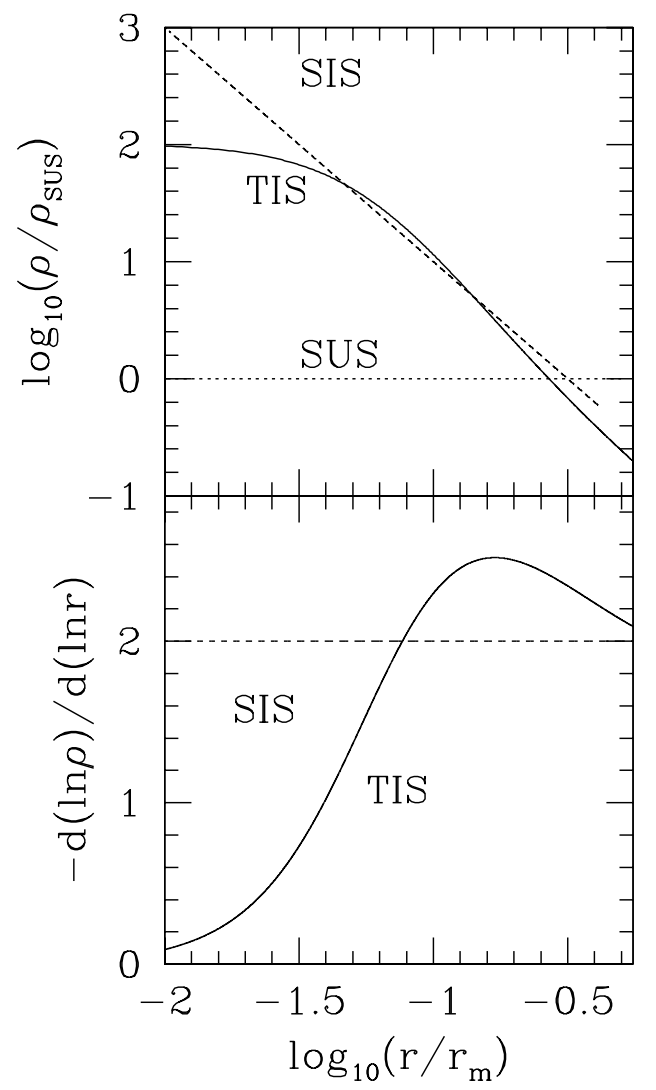

Figure 3. (upper panel) The postcollapse equilibrium density profile of a spherical top-hat density perturbation versus the radius in units of the radius of the tophat at maximum expansion for the minimum-energy TIS (solid line), the singular isothermal sphere (SIS, dashed line) and the uniform sphere approximation (SUS, dotted line). All densities are plotted in units of $18 \pi^{2} \rho\left(t_{\text {coll }}\right)$, the postcollapse density of the SUS approximation. (lower panel) The logarithmic slope of our minimum-energy TIS profile versus radius as compared to the constant slope of -2 for the SIS profile.

of infall is converted primarily into the thermal energy of the shock-compressed gas, with only a small portion remaining as kinetic energy of subsonic, postshock infall. By analogy with this infall solution, therefore, we identify the boundary pressure $p_{t}$ in the case of our TIS solution, not as a fixed external pressure which acts on the top-hat boundary throughout its collapse and virialization, but rather as something like the instantaneous post-shock pressure in the infall solution. As such, it has a physical origin in the conversion of the original energy of the collapsing top-hat, itself, from potential energy at maximum expansion into kinetic energy of infall during collapse and, finally, into thermal energy of the post-shock gas, always conserving the original energy $E$ of the top-hat.

The final size of the sphere after collapse and virialization is related to the size of the parent top-hat density fluctuation at the epoch of turnaround by a collapse factor $\eta$ defined by

$\eta=\frac{r_{t}}{r_{m}}$

Using equations $(69)$ and $(63)$, it is easy to see that

$\eta=-\frac{5}{3} \lambda_{E}=\frac{5}{2} \frac{\alpha\left(\zeta_{t}\right)-2}{\alpha\left(\zeta_{t}\right)} \frac{\zeta_{t}}{\tilde{M}\left(\zeta_{t}\right)}$

A plot of $\eta\left(\zeta_{t}\right)$ versus $\zeta_{t}$ is shown in Figure 4 . For the minimum-energy solution, using $\zeta=\zeta_{t}=29.4$ in equation 70 yields $\eta=0.554$, i.e. $r_{t}=0.554 r_{m}$. This value of $\eta$ derived for the minimum-energy TIS solution above is similar to the value usually cited for the postcollapse, virialized object in the SUS approximation, for which $r_{\mathrm{vir}}=r_{m} / 2$, corresponding to $\eta_{S U S}=0.5$. It is somewhat larger than the value for the limiting case of a singular isothermal sphere ("SIS"), for which $\eta=0.4167$.

The radius $r_{t}$ of the TIS solution is encountered by the outer boundary of the collapsing top-hat at a time somewhat 


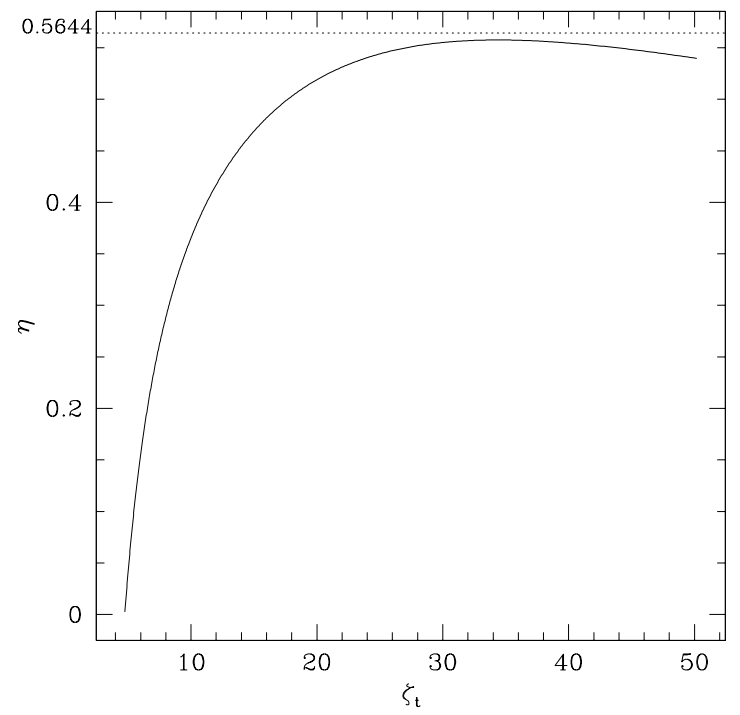

Figure 4. The collapse factor relating the radius of the initial top-hat at its point of maximum expansion to its radius after collapse and virialization, $\eta \overline{=} r_{t} / r_{m}$, as function of the truncation radius $\zeta_{t}$. The dotted line indicates the value of $\eta$ given by the self-similar infall solution (see $\$$ 开).

earlier than the time $t_{\text {coll }}$ of infinite density in the top-hat solution. We shall refer to the time at which the radius of the collapsing top-hat equals $r_{t}$ as $t_{\text {cross }}$, to distinguish it from $t_{\text {coll }}$. At time $t_{\text {cross }}$, the top-hat exact solution (1) yields an overdensity of $\delta=\delta_{\text {cross }} \approx 100$, while the extrapolated linear solution at this time yields $\delta_{L}=\delta_{L, \text { cross }} \approx 1.52$. If we assume that the TIS forms instantaneously at $t=t_{\text {cross }}$, then its mean density corresponds to a mean overdensity $\bar{\delta}=\bar{\delta}_{\text {cross }} \approx 100$ when compared with the background density at $z_{\text {cross }}$, and corresponds to a mean overdensity $\bar{\delta}=\bar{\delta}_{\text {coll }} \approx 130$ when compared with the background density at $z_{\text {coll }}$. This latter value differs somewhat from the conventional value of $18 \pi^{2} \approx 178$, which is found in the SUS approximation.

In applications of the top-hat model involving the Press-Schechter approximation, it is customary to identify the characteristic time of formation of objects of a given mass in terms of $\delta_{c}$ in equation (2). Since $\delta_{L \text {, cross }}<\delta_{c}$, it may be appropriate to replace $\delta_{c}$ in such applications by the value $\delta_{L, \text { cross }} \approx 1.52$, implying that the number of objects formed at any epoch with a mass greater than some value will be somewhat higher for the TIS solution than previously assumed in applications of the Press-Schechter approximation. We note that comparisons of the predictions of the Press-Schechter approximation for the mass function of virialized haloes at any epoch with the results of $N$-body simulations of cosmic structure formation from scale-free initial conditions have sometimes indicated better agreement if the standard value of $\delta_{c}=1.69$ is replaced by a lower value of $\delta_{c}$ (e.g. Efstathiou and Rees 1988; Carlberg and Couchman 1989; Klypin et al. 1995; Bond and Myers 1996; Cen 1998). Perhaps our TIS result that $\delta_{L, \text { cross }} \approx 1.52$ is part of the explanation for this.

In order to check our derivation of the unique TIS solution described above and confirm the validity of the minimumenergy argument on which it is based, we take a second approach to the same problem, in the following section. In this second approach we match the properties of the final TIS equilibrium configuration to those of the post-shock gas in the well-known, frequently applied, self-similar, cosmological, spherical infall solution (Bertschinger 1985). As we shall see, this approach not only confirms our derivation above, but also provides a dynamically self-consistent origin for the final equilibrium state of the TIS.

\section{APPLICATION OF THE SELF-SIMILAR SPHERICAL INFALL SOLUTION}

The description of the postcollapse virial equilibrium of a top-hat density perturbation as a TIS with the same total mass and energy as the parent top-hat is not unique without the use of additional information which establishes the radius, the temperature, or the boundary pressure. We have argued above that a natural outcome of the dynamical relaxation of the collapsed top-hat is that unique TIS which makes its energy $E$ correspond to a minimum-energy solution at fixed boundary pressure. This, in turn, establishes what the value of $p_{t}$ is for the TIS which results from the collapse of a given top-hat, and, thereby, fixes the unique TIS solution. To test this anzatz, we shall now place our top-hat density perturbation and its collapse and virialization in a dynamically self-consistent context, by comparing with the closely related problem of the spherical infall which results when a point-mass perturbation is added to a matter-dominated Einstein-de Sitter universe with initially 
uniform density, for which an exact solution is possible. This latter problem represents the simplest kind of generalization of a uniform top-hat initial condition which can lead to an interruption of the collapse of the entire perturbation to infinite density at a finite time, due to the radial nonuniformity of the initial conditions. As such, this problem can be used to provide some insight into how inhomogeneities in a collapsing top-hat might halt its collapse prior to its reaching infinite density, causing it to thermalize the kinetic energy of collapse and relax to an equilibrium configuration. The spherical symmetry of this point-mass perturbation problem, moreover, means that one can identify a corresponding top-hat perturbation for the mass interior to any spherical shell in the point-mass perturbation problem, by defining the top-hat which contains the same total mass and energy inside the same size sphere at the same initial moment. Hence, a quantitative comparison of the solution of this problem and a suitably chosen, matching top-hat problem should be possible. We describe this in what follows.

\subsection{The self-similar spherical infall solution}

Consider the self-similar spherical infall solution for the flow which results when a point-mass perturbation is introduced at some point in an otherwise unperturbed Einstein-de Sitter universe (Bertschinger 1985). For a collisional fluid, this solution involves an accretion shock which occurs at radius $r_{S}=\lambda_{S} r_{t a}$, where $r_{t a}$ is the radius of that spherical shell of matter surrounding the initial perturbation which is just turning around at any epoch $t$ and where $\lambda_{S}$ is a constant. For an ideal gas with ratio of specific heats $\gamma=5 / 3$, the constant $\lambda_{S} \approx 0.34$. As we shall describe in more detail below, it is appropriate for us approximately to identify the region of this self-similar infall which is bounded by the accretion shock at radius $r_{S}$ with our postcollapse equilibrium sphere. In order to do this, we must make the appropriate correspondence between the top-hat perturbation in our initial conditions and the "matching" top-hat in the infall solution which results when we add the initial point-mass perturbation to the unperturbed gas in a finite sphere so as to encompass the same mass in the infall solution as in our initial top-hat. The correct "matching" of the two solutions must ensure that the spherical shell which is just reaching the shock in the infall solution at some time $t$ was following the same trajectory as the outer boundary of our top-hat perturbation at all times prior to the time it encounters the shock at $r_{S}$. We outline this "matching" solution below. We argue, further, that this collisional fluid solution should apply equally well to the case of a TIS in a gas of cold, collisionless dark matter or any combination of fluid with $\gamma=5 / 3$ and cold, collisionless gas, as follows. Interestingly enough, for the same kind of perturbation in an unperturbed, purely collisionless, initially cold gas (e.g. cold dark matter), the self-similar solutions of Bertschinger (1985) and Fillmore and Goldreich (1984) yield an inner region of shell crossings, bounded by the outermost caustic located at $r_{c, 1}=\lambda_{c, 1} r_{t a}$, where $\lambda_{c, 1} \approx 0.36$. As a result, the outer boundaries of the shocked gas and of the region of the shell-crossings and caustics in the collisionless dark matter are almost coincident, and their respective mass distributions are unbiased with respect to each other. In that case, we can focus in what follows on the Bertschinger (1985) solution for a collisional fluid with $\gamma=5 / 3$ and $\Omega=1$, but bear in mind that our final result for the TIS applies equally well to a collisionless dark matter halo or to a combination of the two, as well, as long as $\Omega=1$.

Following Bertschinger (1985), we define the dimensionless variable $\lambda=r / r_{t a}(t)$, where $r_{t a}$ is the radius of the shell that is just turning around at the time $t$. The fluid variables pressure $p$, mass density $\rho$, and mass $m(r)$ interior to radius $r$, are non-dimensionalized using the following definitions of dimensionless pressure $P$, density $D$ and mass $M$ interior to dimensionless radius $\lambda=r / r_{t a}$ :

$p(r, t)=\rho_{b}\left(\frac{r_{t a}}{t}\right)^{2} P(\lambda), \quad \rho(r, t)=\rho_{b} D(\lambda), \quad m(r, t)=\frac{4}{3} \pi \rho_{b} r_{t a}^{3} M(\lambda)$,

where $\rho_{b}$ is the mean, unperturbed background density at time $t$. Inserting these definitions into the fluid conservation partial differential equations leads to a set of ordinary differential equations. These equations, together with strong, adiabatic shock jump boundary conditions, the exact, pressure-free infall solution for the flow exterior to the shock and the assumption that the energy of the gas bounded by the shock is the same as the initial energy of the same mass when it was outside the shock, describe a self-similar infall solution with an accretion shock. Bertschinger (1985) presented detailed results of his numerical solution of these equations both graphically and in tabular form. However, since we shall need a finer grid of solution values than is tabulated there, we have re-integrated the resulting equations ourselves for the current paper, using the stiff equation solver RADAU5 by E. Hairer and G. Wanner (Hairer, Norsett \& Wanner 1996).

The shock occurs at fixed $\lambda=\lambda_{S} \equiv r_{S} / r_{t a}$ and propagates according to

$r_{S}(t)=\lambda_{S} r_{t a}(t) \propto t^{8 / 9}$.

The mass inside the shock is determined by:

$m\left(r_{S}, t\right)=\frac{4}{3} \pi \rho_{b} r_{t a}^{3}(t) M\left(\lambda_{S}\right)$,

The self-similar infall solution yields, for $\gamma=5 / 3$ :

$\lambda_{S}=0.338976, \quad M\left(\lambda_{S}\right)=3.78759, \quad P\left(\lambda_{S}\right)=9.73563, \quad D\left(\lambda_{S}\right)=16.5341$.

(C) 0000 RAS, MNRAS 000, 000-000 


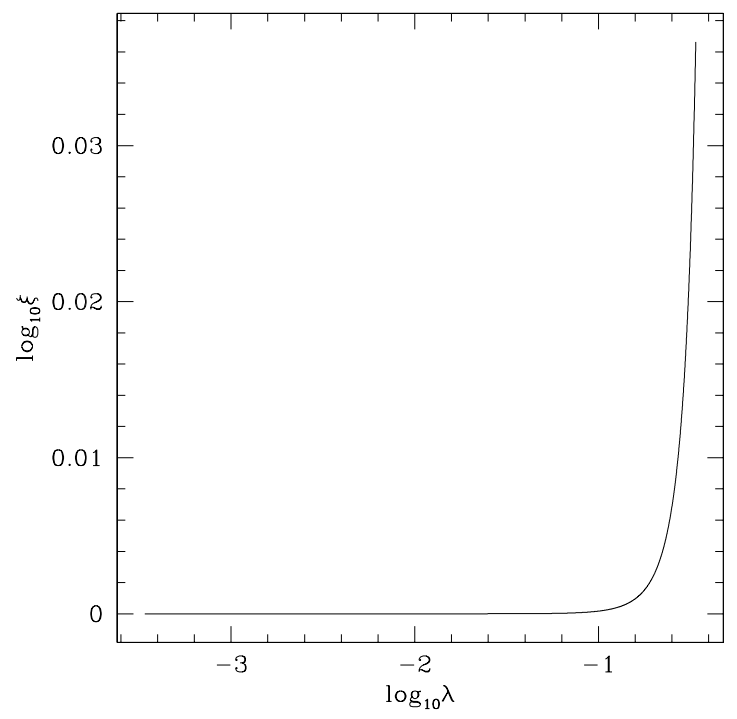

Figure 5. The ratio of pressure force to gravitational force inside the accretion shock in the self-similar infall solution. A value $\xi=1$ implies hydrostatic equilibrium. The shock is located at $\log _{10} \lambda_{S}=-0.469831$.

The mean overdensity inside the shock relative to the mean unperturbed background density is constant in time, and has a value

$\bar{\rho}_{S}=\frac{m\left(r_{S}, t\right)}{\frac{4}{3} \pi r_{S}^{3} \rho_{b}}=\frac{M\left(\lambda_{S}\right)}{\lambda_{S}^{3}}=97.24$

Consider the post-shock gas in this self-similar infall solution. Strictly speaking, this postshock flow is neither isothermal nor in hydrostatic equilibrium. As we shall see, however, the variation of temperature with radius is rather weak, so isothermality is a reasonable approximation and the post-shock gas is in approximate hydrostatic equilibrium, as well. We shall demonstrate this as follows. If the gas is in hydrostatic equilibrium, it must satisfy the equation

$\frac{1}{\rho} \frac{d p}{d r}=-\nabla \phi$.

Let us define a dimensionless ratio $\xi$,

$\xi \equiv \frac{1}{\rho} \frac{d p}{d r} /(-\nabla \phi)$

which measures the degree to which hydrostatic equilibrium is obtained at any radius $r$, so that $\xi=1$ if equilibrium occurs. In order to demonstrate how close the exact solution is to hydrostatic equilibrium inside the shock, we rewrite $\xi$ in terms of the dimensionless fluid variables, according to

$\xi(\lambda)=\frac{9}{2}\left[\frac{\lambda^{2} P^{\prime}(\lambda)}{M(\lambda) D(\lambda)}\right]$

We use the exact numerical solution to plot $\xi(\lambda)$ in Figure Ap Aparently, the postshock gas is always close to hydrostatic, with a maximum departure of $\xi$ from unity of 9 per cent just behind the shock, where the gas still has a small infall velocity and the pressure is a little lower than the value required to balance gravity.

The approximation of equilibrium becomes perfect as we approach the origin. We can demonstrate this analytically, using the simple asymptotic scalings of the fluid variables with distance there. In terms of $\lambda$, the solution obeys the following scalings at small $\lambda$ :

$P=\tilde{P}(\lambda) \lambda^{-5 / 2}, \quad D=\tilde{D}(\lambda) \lambda^{-9 / 4}, \quad M=\tilde{M}(\lambda) \lambda^{3 / 4}$,

where $\tilde{P}, \tilde{D}$ and $\tilde{M}$ are only weakly dependent on $\lambda$ for small $\lambda, \tilde{P}(0)=2.40676, \tilde{D}(0)=2.60173$, and $\tilde{M}(0)=10.4069$. Inserting equation (79) into equation (78) for $\xi(\lambda)$ shows that $\xi$ is independent of $\lambda$ as $\lambda$ approaches 0 , with $\xi(0)=1$.

The postshock gas is also not far from isothermal, despite the strong increase of pressure and density towards the centre. The volume-averaged temperature interior to the shock radius is related to the immediate postshock temperature $T_{S}$ according to

$\frac{\langle T\rangle_{V}}{T_{S}}=3 \lambda_{S}^{-3} \int_{0}^{\lambda_{S}} \frac{P(\lambda) / P\left(\lambda_{S}\right)}{D(\lambda) / D\left(\lambda_{S}\right)} \lambda^{2} d \lambda$. 
According to the scaling laws in equation $(79)$, valid at small radii and approximately correct throughout much of the postshock region, temperature varies with radius only as $r^{-1 / 4}$. In terms of the scaling laws,

$\frac{\langle T\rangle_{V}}{T_{S}} \approx \frac{12}{11} \lambda_{S}^{-1 / 4} \frac{\tilde{P}(0) / P\left(\lambda_{S}\right)}{\tilde{D}(0) / D\left(\lambda_{S}\right)} \approx 2.25$,

while the exact solution yields

$\frac{\langle T\rangle_{V}}{T_{S}}=1.386$

which is remarkably close to unity.

In short, the self-similar infall solution indicates that the dynamically self-consistent evolution of an uncompensated, spherically-symmetric, positive, cosmological density perturbation in a cold gas leads to continuous infall and the creation of a continuous sequence of spheres of collapsed gas which are, at any instant, in a state which is close to that of an isothermal sphere in hydrostatic equilibrium, bounded by the accretion shock. In what follows, we identify the particular self-similar, shock-bounded sphere in this solution which instantaneously corresponds to the postcollapse, virialized sphere which forms as a result of the equivalent top-hat perturbation.

\subsection{Matching the post-shock gas in the infall solution with the postcollapse TIS of the top-hat}

In what follows, we attempt to find the TIS solution for the postcollapse virial equilibrium of a top-hat density perturbation which most closely matches the characteristics of the accretion-shock-bounded sphere in the self-similar spherical infall solution when the mass and energy inside the latter sphere equal the mass and energy of the top-hat. We require that the trajectory of the top-hat outer boundary be the same as that of the immediate postshock fluid element in the infall solution during the preshock infall of the latter. This fixes the radius of the shock and the time at which the shock encloses a mass equal to that of the corresponding top-hat. By comparing this shock radius with the radius of the immediate postshock fluid element at its point of maximum expansion, we shall derive the effective collapse factor for the corresponding top-hat from its maximum expansion to its postcollapse virial equilibrium state, by identifying the shock radius $r_{S}$ as the radius $r_{t}$ of the matching TIS. As we shall see, this infall shock radius in almost precisely equal to the radius derived previously for the minimum-energy TIS solution, although it is necessary to make a tiny downward adjustment of the value of $r_{t}$ relative to $r_{S}$ in order to match a TIS solution with the same total mass and energy. In particular, an isothermal sphere with exactly the same mass and energy as this shock-bounded sphere in the infall solution can only be in hydrostatic equilibrium (i.e. a solution of the Lane-Emden equation) if its radius is a tiny bit smaller than the shock radius in the infall solution. We shall make an approximation, therefore, to determine the actual radius $r_{t}$, slightly below $r_{S}$, of the equilibrium TIS solution which most closely matches the shock-bounded sphere in the infall solution, as follows. As we shall see, the resulting TIS is not far from our TIS solution derived above from the minimum-energy argument, so we can be confident that our minimum-energy TIS solution has a sound physical basis. We begin by solving for the shock radius $r_{S}$ and time $t_{\text {cross }}$ at which the collapsing outer boundary of the top-hat reaches the radius of the shock in the infall solution, when the gas enclosed by the shock in that solution contains the same mass $M_{0}$ and energy $E$ as the top-hat.

The total mass of the top-hat we consider is:

$M_{0}=\frac{4 \pi}{3} r_{m}^{3}\left(1+\delta_{m}\right) \rho_{b}\left(t_{m}\right)$.

We require that the masses of the top-hat and the post-shock gas in the self-similar infall solution be equal at the time when the outer radius of the top-hat crosses the radius of the shock. This yields

$M_{0}=m\left(r_{S}, t_{\mathrm{cross}}\right)=\frac{4}{3} \pi \rho_{b}\left(t_{\mathrm{cross}}\right) r_{\text {ta }}^{3}\left(t_{\mathrm{cross}}\right) M\left(\lambda_{S}\right)$,

and, therefore,

$r_{t a}\left(t_{\text {cross }}\right)=\left[\frac{M_{0}}{M\left(\lambda_{S}\right)} \frac{3}{4 \pi \rho_{b}\left(t_{\text {cross }}\right)}\right]^{1 / 3}$,

where

$\rho_{b}(t)=\left(6 \pi G t^{2}\right)^{-1}, \quad \rho_{b}\left(t_{\text {cross }}\right)=\rho_{b 0}\left(1+z_{\text {cross }}\right)^{3}$,

and $\rho_{b 0}=3 H_{0}^{2} / 8 \pi G$, the mean background density at present. Combining equations (83) - (86), we can write

$\left[\frac{r_{t a}\left(t_{\text {cross }}\right)}{t_{\text {cross }}}\right]^{2}=\frac{3}{2} \frac{(36 \pi)^{1 / 3} G}{\left[M\left(\lambda_{S}\right)\right]^{2 / 3}} M_{0}^{2 / 3}\left(1+z_{\text {cross }}\right) \rho_{b 0}^{1 / 3}$,

and

(C) 0000 RAS, MNRAS 000, 000-000 
$r_{m}=\left(\frac{3}{4 \pi}\right)^{1 / 3} \frac{\lambda_{S}}{\eta\left[M\left(\lambda_{S}\right)\right]^{1 / 3}} M_{0}^{1 / 3}\left(1+z_{\mathrm{cross}}\right)^{-1} \rho_{b 0}^{-1 / 3}$

The radius of the shock wave at $t_{\text {cross }}$ is, using equations (2) and 85, given by

$$
\begin{aligned}
r_{S}\left(t_{\text {cross }}\right) & =\lambda_{S} r_{t a}\left(t_{\text {cross }}\right)=\frac{\lambda_{S}}{\left[M\left(\lambda_{S}\right)\right]^{1 / 3}}\left(1+\delta_{m}\right)^{1 / 3}\left(\frac{t_{\text {cross }}}{t_{m}}\right)^{2 / 3} r_{m} \\
& =\frac{\lambda_{S}}{\left[M\left(\lambda_{S}\right)\right]^{1 / 3}}\left(1+\delta_{m}\right)^{1 / 3} \frac{\delta_{L, \text { cross }}}{\delta_{L m}} r_{m}=0.36244 \delta_{L, \text { cross }} r_{m},
\end{aligned}
$$

where $\delta_{L, \text { cross }}$ is the linear density contrast corresponding to the time $t_{\text {cross }}$. Expressed in terms of $r_{m}$, this yields,

$r_{S}\left(t_{\text {cross }}\right)=\eta_{S} r_{m}$,

where $\eta_{S}=0.564393$ Bertschinger 1985).

Our goal here was to use the shock radius $r_{S}\left(t_{\text {cross }}\right)$ to establish $r_{t}$, the size of our postcollapse equilibrium TIS, by equating $\eta$ in equation (69) with $\eta_{S}$ in equation (90). This value of $\eta_{S}$ is extremely close to the value of $\eta=0.554$ derived above for the unique minimum-energy TIS solution for the postcollapse equilibrium of the top-hat. Such an agreement is remarkable and can hardly be a coincidence. We take this as a strong confirmation of the validity of that minimum-energy solution. However, as mentioned earlier, we also note that this tiny difference between $\eta=0.564$ and $\eta=0.554$ is nevertheless enough to put the isothermal sphere with mass $M$, energy $E$ and radius $r_{t}=r_{S}$ ever so slightly beyond the boundary of allowed solutions of the Lane-Emden equations, according to Figure 4 and equation (70). In other words, since $\lambda_{E}=-(3 / 5) \eta_{S}=-0.338636<-0.335$ in this case, such an isothermal sphere is just slightly too large to be an equilibrium TIS. In what follows we shall refine this estimate of the TIS radius $r_{t}$ so that it departs from $r_{S}$ only minimally but nevertheless preserves the physical basis in terms of the self-similar infall solution.

\subsubsection{The TIS solution whose size is closest to the shock radius in the infall solution}

The significance of the remarkably close agreement between the radius $r_{S}\left(t_{\text {cross }}\right)$ derived above and the radius $r_{t}$ of the minimum-energy TIS for the same parent top-hat is made clear by the curve of $\eta$ versus $\zeta_{t}$ in Figure 7 which shows that $\eta=0.554$, the value for $\zeta_{t}=29.4$, the minimum-energy TIS solution value is close to the maximum value of $\eta$ for all values of $\zeta_{t}$, while $\eta_{S}=0.564$ is only just above the curve. Since the postshock flow is only approximately isothermal and hydrostatic it is reasonable to suppose that the final relaxation to equilibrium keeps the total energy and mass constant but allows the radius to shrink slightly, just enough to relax to a stable equilibrium TIS. The simplest way to refine the value of $r_{t}$ (or, equivalently, of $\eta$ ) downward from $r_{S}$ ( or $\eta_{S}$ ) so as to make an equilibrium TIS solution possible, therefore, is just to take $\eta=\eta_{\max }$, the maximum value of $\eta$ versus $\zeta_{t}$ and, hence, the allowed value which is closest to $\eta_{S}$. This yields the estimate $\eta_{1}=\eta_{\max }=0.558$, for which $\zeta_{t}=\zeta_{t, 1}=34.2$ and $\alpha=\alpha_{1} \equiv 3.66$. This $\eta_{\max }$ solution corresponds exactly to the unique stable equilibrium TIS for which $\lambda_{E}=-0.335$ and $\mathcal{R}=708.61$. This value of $\eta=\eta_{1}$ differs from $\eta_{S}$ by only 1.3 per cent. With this choice of $\eta_{1}$, the TIS solution is extremely close to that from the minimum-energy solution, with $\eta_{1}$ differing by only 0.7 per cent, $\alpha$ and $T$ for the two solutions differing by less than 2 per cent for each, and $\zeta_{t}$ (i.e. the core radius $r_{0}$ ) by only 17 per cent.

This agreement between our minimum-energy TIS solution and the infall accretion-shock size matching TIS solution confirms the validity of our minimum-energy TIS solution. We note, however, that even the very small difference found between the two TIS solutions is just enough to place the size-matching solution on the boundary, described above, of gravothermal catastrophe. Apparently, self-similar spherical infall leads naturally to the formation of a shock-bounded sphere which is close to that of an isothermal sphere on the edge of the gravothermal catastrophe regime.

\section{DISCUSSION AND SUMMARY}

\subsection{Summary of the minimum-energy TIS solution}

We have argued above that a cosmological top-hat density perturbation in an Einstein-de Sitter universe which virializes following its collapse can be described self-consistently by a unique TIS solution which conserves the energy of the initial top-hat and is bounded by a pressure which makes that energy a minimum. The temperature derived in this way for the sphere in virial and hydrostatic equilibrium is larger by a factor of approximately two than the value previously derived by satisfying energy conservation and the virial theorem for a postcollapse sphere of uniform density for which the surface pressure term is neglected. The origin of the boundary pressure term in the TIS solution can be understood self-consistently by comparison with the well-known self-similar, spherical infall solution of Bertschinger(1985) for the problem of the collapse of matter onto a point-mass perturbation added to a uniform, cosmologically expanding background. In the latter problem, spherical mass shells surrounding the point mass decelerate relative to the mean cosmic expansion, reach a maximum radius 
and collapse back toward the center supersonically, unaffected by pressure forces, following a trajectory identical to that of the outer boundary of a top-hat perturbation which encompasses the same total mass and energy. Unlike the collapse of the top-hat, however, the self-similar infall is interrupted by a strong accretion shock which decelerates the flow and thermalizes the kinetic energy of infall, allowing the postshock gas to settle subsonically into a state which is approximately hydrostatic and isothermal. By comparing the shock-bounded sphere in this self-similar infall solution to the unique minimum-energy TIS solution which we derived here by a completely independent line of reasoning, we have been able to confirm the validity of the TIS solution while explaining its dynamical origin and the nature of the boundary pressure. The boundary pressure, in short, is closely related to the presence of the accretion shock in the self-similar infall solution. While the TIS boundary pressure is not identical to the instantaneous postshock pressure in the infall solution (it is slightly higher), the TIS boundary pressure corresponds to the slightly adjusted postshock pressure required to transform the quasi-hydrostatic postshock flow into an exact hydrostatic equilibrium while conserving the total energy inside the sphere. We have also used the properties of the self-similar infall solution in the case in which both collisionless dark matter and a collisional fluid are present to argue that we can apply our TIS solution to either component or both. For the collisionless component, velocity dispersion takes the place of temperature. If both components are present, then the density of each component $i, \rho_{i}$, is simply proportional to the total, $\rho_{i}=\rho_{\text {tot }} \Omega_{i}$, where $\Omega_{i}$ is the universal density parameter for component $i$.

The TIS solution for the postcollapse equilibrium of a top-hat of mass $M_{0}$ which would collapse at epoch $z_{\text {coll }}$ if its collapse were not interrupted by virialization depends upon $M_{0}, z_{\text {coll }}, h$, the mean total background density $\rho_{b, 0}\left(\right.$ where $\rho_{b, 0} \propto h^{2}$ ), and the ratio $\zeta_{t}$ of truncation radius $r_{t}$ to core radius $r_{0}$, according to

$$
\begin{aligned}
T & \propto \frac{\alpha}{\alpha-2} M_{0}^{2 / 3}\left(1+z_{\mathrm{coll}}\right) h^{2 / 3}, \\
v_{c} & \propto \eta^{-1 / 2} M_{0}^{1 / 3}\left(1+z_{\mathrm{coll}}\right)^{1 / 2} h^{1 / 3}, \\
r_{m} & \propto M_{0}^{1 / 3}\left(1+z_{\mathrm{coll}}\right)^{-1} h^{-2 / 3}, \\
\rho_{0} & \propto \frac{\zeta_{t}^{3}}{\eta^{3}} \frac{1}{\tilde{M}_{t}} \rho_{b 0}\left(1+z_{\mathrm{coll}}\right)^{3},
\end{aligned}
$$

where $\alpha$ and $\tilde{M}_{t}$ depend only on $\zeta_{t}$ and where $h$ is the Hubble constant in units of $100 \mathrm{~km} \mathrm{~s}^{-1} \mathrm{Mpc}^{-1}$. The radii $r_{t}=\eta r_{m}$ and $r_{0}=r_{t} / \zeta_{t}$ have the same scaling with $M_{0}, 1+z_{\text {coll }}$, and $h$ as does $r_{m}$.

The value we have derived above for $\zeta_{t}$ for our minimum-energy TIS solution is $\zeta_{t}=29.4$. All of the parameters of this

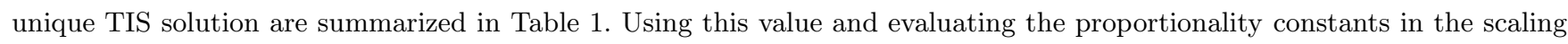
laws (91) - 94), the scaling laws become the following:

$$
\begin{aligned}
r_{m} & =\left(\frac{8 G}{9 \pi^{2}}\right)^{1 / 3} M_{0}^{1 / 3}\left(1+z_{\mathrm{coll}}\right)^{-1} H_{0}^{-2 / 3}=338.3\left(\frac{M}{10^{12} M_{\odot}}\right)^{1 / 3}\left(1+z_{\mathrm{coll}}\right)^{-1} h^{-2 / 3} \mathrm{kpc}, \\
r_{t} & =\eta r_{m}=187.4\left(\frac{M}{10^{12} M_{\odot}}\right)^{1 / 3}\left(1+z_{\mathrm{coll}}\right)^{-1} h^{-2 / 3} \mathrm{kpc}, \\
T & =\frac{(3 \pi)^{2 / 3}}{5} \frac{\alpha}{\alpha-2} \frac{m_{p}}{k_{B}} G^{2 / 3} \mu M_{0}^{2 / 3}\left(1+z_{\mathrm{coll}}\right) H_{0}^{2 / 3}=1.328 \times 10^{6} \mu\left(\frac{M_{0}}{10^{12} M_{\odot}}\right)^{2 / 3}\left(1+z_{\mathrm{coll}}\right) h^{2 / 3} \mathrm{~K} \\
\sigma^{2} & =\frac{k_{B} T}{m}=\frac{(3 \pi)^{2 / 3}}{5} \frac{\alpha}{\alpha-2} G^{2 / 3} M_{0}^{2 / 3}\left(1+z_{\mathrm{coll}}\right) H_{0}^{2 / 3}=1.096 \times 10^{4}\left(\frac{M_{0}}{10^{12} M_{\odot}}\right)^{2 / 3}\left(1+z_{\mathrm{coll}}\right) h^{2 / 3} \mathrm{~km}^{2} \mathrm{~s}^{-2} \\
v_{c} & =\left(\frac{G M_{0}}{r_{t}}\right)^{1 / 2}=151.4\left(\frac{M_{0}}{10^{12} M_{\odot}}\right)^{1 / 3}\left(1+z_{\mathrm{coll}}\right)^{1 / 2} h^{1 / 3} \mathrm{~km} / \mathrm{s}, \\
r_{0} & =\frac{r_{t}}{\zeta_{t}}=6.37\left(\frac{M_{0}}{10^{12} M_{\odot}}\right)^{1 / 3}\left(1+z_{\mathrm{coll}}\right)^{-1} h^{-2 / 3} \mathrm{kpc}, \\
\rho_{0} & =6 \pi^{2}\left(\frac{b_{T}}{5}\right)^{3} \tilde{M}_{t}^{2}\left(1+z_{\mathrm{coll}}\right)^{3} \rho_{b 0}=1.796 \times 10^{4}\left(1+z_{\mathrm{coll}}\right)^{3} \rho_{b 0}=3.376 \times 10^{-25}\left(1+z_{\mathrm{coll}}\right)^{3} h^{2} \mathrm{~g} / \mathrm{cm}^{3}
\end{aligned}
$$

The mean density inside the TIS is

$\bar{\rho}=3 \frac{\tilde{M}\left(\zeta_{t}\right)}{\zeta_{t}^{3}} \rho_{0}=7.25 \times 10^{-3} \rho_{0}=18 \pi^{2}\left(\frac{b_{T}}{5}\right)^{3} \frac{\tilde{M}_{t}^{3}}{\zeta_{t}^{3}} \rho_{b 0}\left(1+z_{\text {coll }}\right)^{3}=130.5 \rho_{b 0}\left(1+z_{\text {coll }}\right)^{3}$.

Together with a numerical solution of the Lane-Emden equation in dimensionless form for the sphere with parameters given in Table 1, shown in Figures 3 and 6, these scaling laws provide the complete description necessary to apply our TIS solution to some cosmological problem.

For some purposes, an approximate fitting formula for the numerical solution of the Lane-Emden equation for isothermal spheres, equations (29) and (30), is convenient. Although we have worked exclusively with the actual numerical solution of equation (29) throughout this paper, so as to minimize our errors, we shall now provide such a fitting formula which is useful 
Table 1. Summary of the minimum-energy TIS solution

\begin{tabular}{ll} 
Quantity & Value \\
\hline$\zeta_{t}=\frac{r_{t}}{r_{0}} \ldots \ldots \ldots$ & 29.40 \\
$\alpha\left(\zeta_{t}\right)=\frac{\bar{p}}{p_{t}} \ldots$ & 3.73 \\
$\tilde{M}\left(\zeta_{t}\right) \ldots \ldots \ldots$ & 61.48 \\
$b_{T}=\frac{T_{T I S}}{T_{S U S} \ldots}$ & 2.16 \\
$\eta=\frac{r_{t}}{r_{m}} \ldots \ldots \ldots$ & 0.554 \\
$\frac{r_{T I S}}{r_{S U S} \ldots \ldots \ldots \ldots}$ & 1.11 \\
$\delta_{L, \text { cross }} \ldots \ldots \ldots$ & 1.52 \\
$\delta\left(t_{\mathrm{cross}}\right) \ldots \ldots$. & 97.2 \\
$\delta\left(t_{\mathrm{coll}}\right) \ldots \ldots \ldots$ & 130.5 \\
$\frac{\rho_{t} \ldots \ldots \ldots \ldots \ldots}{\rho_{0} \ldots \ldots \ldots \ldots . . .}$ & $1.95 \times 10^{-3}$ \\
$\mathcal{R} \ldots \ldots \ldots \ldots \ldots$ & 514 \\
$\frac{\rho_{0}}{\rho_{b, \text { coll }} \ldots \ldots \ldots \ldots .}$ & $1.80 \times 10^{4}$ \\
$\frac{\rho_{t}}{\rho_{b, \mathrm{coll}}} \ldots \ldots \ldots \ldots$ & 35.0 \\
\hline
\end{tabular}

as a good approximation over a wider range of radii than has been presented in previous treatments in the literature. We adopt the form

$\tilde{\rho}=\frac{\rho}{\rho_{0}}=\frac{A}{a^{2}+\zeta^{2}}-\frac{B}{b^{2}+\zeta^{2}}$

with the parameters $A, B, a$, and $b$ sensibly chosen (Natarajan and Lynden-Bell 1997; henceforth, NL97). In the Appendix, we briefly describe the method used to obtain such a solution, adjusted to the problem at hand. For the purposes of this paper a very good approximation is that of equation (103) with the following parameters:

$\left(A, a^{2}, B, b^{2}\right)_{T I S}=(21.38,9.08,19.81,14.62)$

which differs from that in NL97 and is a better fit over a larger range of radii than theirs (see Appendix A1). It fits the exact solution within a fractional error of 3 per cent for $0<\zeta<40$, which is a range that fully encompasses that of our final TIS solution, with a perfect match to the exact solution at the boundary $\zeta=\zeta_{t}=29.4$.

\subsection{Comparison of the minimum-energy TIS solution and the uniform sphere and singular isothermal sphere approximations}

The temperature derived here by the minimum-energy TIS solution for the postcollapse sphere in virial and hydrostatic equilibrium which follows top-hat collapse is a factor of approximately two larger than the value previously derived by satisfying energy conservation and the virial theorem for a postcollapse sphere of uniform density for which the surface pressure term is neglected, as summarized in $\S$. In particular, if we write $T_{T I S}=b_{T} T_{S U S}$, then $b_{T}=\alpha /(\alpha-2)=2.16$. We shall address the question of the significance of this revision of the postcollapse virial temperature below. The size of the TIS sphere, given in terms of the radius $r_{m}$ of the top-hat at maximum expansion according to $r_{t}=0.554 r_{m}$, is actually not far from the size of the SUS sphere, $r_{\text {vir }}=r_{m} / 2$. However, in terms of the TIS core radius $r_{0}$, the truncation radius is large, $r_{t} / r_{0}=29.4$, implying that the TIS is very far from uniform density. Despite this relatively small core radius, the TIS solution is also quite different from that of a singular isothermal sphere; for the latter, the ratio of the average density to that at the surface is 3 , while we find a value of 3.73 for the nonsingular TIS, and the truncation radius in the singular limit is only $r_{t}=(5 / 12) r_{m}$. Similarly, the correction factor for the virial temperature of the singular isothermal sphere relative to that of the uniform sphere is $b_{T}=3$, as opposed to the value for the TIS which is $b_{T}=2.16$. The central density of the TIS is $1.8 \times 10^{4}$ times larger than the mean density of the background at the collapse epoch for the initial top-hat. Finally, this solution predicts that the top-hat will virialize somewhat earlier than the nominal collapse time of the top-hat, since the outermost mass elements encounter a shock in the infall solution at finite radius. In terms of the usual overdensity of the top-hat predicted by linear theory, this implies a revised value of $\delta_{c}=\left(\delta_{c}\right)_{\text {TIS }}$ where $\left(\delta_{c}\right)_{\text {TIS }} \approx 1.52$, rather than the 
standard value of 1.686 based upon extrapolating the linear growth to the epoch at which the nonlinear top-hat solution predicts infinite density.

\subsection{Comparison of the minimum-energy TIS solution density profile with results of numerical simulation of cosmic structure formation and with observations}

The hydrostatic isothermal sphere has been widely adopted as an equilibrium model for the density profiles of individual cosmic structures. Mass decomposition studies of galaxies based upon rotation curve data, for example, often adopt the density profile $\rho \propto\left[1+(r / a)^{2}\right]^{-1}$, an approximation to a nonsingular isothermal sphere, for the galactic halo (cf. Freeman 1992). As another example, galaxy cluster X-ray brightness profiles are often interpreted in terms of the so-called " $\beta$-model" in which the X-ray emitting gas is an isothermal sphere in hydrostatic equilibrium in the potential well of a virialized dark matter halo, for the purpose of measuring the cluster total mass density and baryon fraction profiles (e.g. Cavaliere and Fusco-Femiano 1976; David, Jones and Forman 1995). In this case, the gas density varies as $\rho \propto\left[1+(r / a)^{2}\right]^{-3 \beta / 2}$, and the value of $\beta=2 / 3$, which corresponds approximately to that of a nonsingular isothermal sphere, is consistent with the data on high temperature X-ray clusters (e.g. Jones \& Foreman 1992; Arnaud and Evrard 1998). These examples of empirical fits are only loosely motivated by theoretical expectations for the dynamical evolution of the objects in question.

Recently, Navarro, Frenk \& White (1996, 1997; NFW) suggested, based upon the results of cosmological N-body simulations of the Cold Dark Matter (CDM) model, that dark matter haloes which condense out of the background can be fit by a universal density profile, of the following form:

$\rho_{N F W}=\frac{\rho_{S}}{\left(r / r_{S}\right)\left(1+r / r_{S}\right)^{2}}$

where the fitting parameters $r_{S}$ and $\rho_{S}$ are related to each other and reflect the different collapse epoch for different mass objects. This NFW profile shares the large-radius asymptotic shape, $r^{-2}$, of an isothermal sphere only for intermediate radii $\left(r / r_{S}>1\right)$, while steepening to $r^{-3}$ at large radii $\left(r / r_{S}>>1\right)$. At small radii $\left(r / r_{S}<<1\right)$, the NFW profile flattens to $r^{-1}$. Unlike the nonsingular isothermal sphere, the NFW profile lacks a finite core. It is natural to ask, therefore, if the apparent differences between the TIS solution presented here for the postcollapse virial equilibrium of a top-hat density fluctuation and the NFW density profile are significant and, if so, what they mean. A full treatment of this question is outside of the scope of this paper. However, we will briefly address the issue as follows.

There are several questions which must be answered in order to compare the TIS and NFW profiles in a meaningful way. First, are they solutions of the same initial value problem? Second, is the NFW halo a state of relaxed, virial equilibrium as the TIS solution is posited to be? Third, is the NFW profile really an accurate fit to the exact results for the dark matter haloes which result from the growth of density fluctuations in a CDM model universe. Fourth, is there a difference between the gas and dark matter profiles expected when objects condense out in the CDM model in general, or when the dark matter profile follows a NFW shape, in particular? Fifth, what do the observations of actual density profiles for cosmologically condensed, virialized structures say regarding the comparison between the TIS solution presented here and the NFW profile?

Regarding the first question, the NFW profile is suggested to be the generic outcome of the condensation of haloes of collisionless dark matter in hierarchical clustering models like CDM, starting from Gaussian random noise density fluctuations. The TIS profile presented here is the outcome of the collapse of an uncompensated spherical top-hat, instead, albeit one in which the presence of some smaller-scale nonuniformity leads to relaxation to a final equilibrium state of finite density. We have argued that a solution very similar to our minimum energy TIS also arises if we consider the self-similar spherical infall solution of Bertschinger (1985), which is already close to isothermal and hydrostatic equilibrium inside the shocked region, and assume that rapid equilibration takes place within this shocked region. In that case, there is a continuous infall which leads to a continuous sequence of TIS solutions of ever larger mass as more mass encounters the accretion shock over time, and the shock represents a boundary which separates the relaxed region in equilibrium inside the shock from the unrelaxed, infalling matter at larger radii. As such, the TIS and NFW profiles do not necessarily arise from identical initial conditions.

The simple spherical top-hat model has been quite successful, however, in characterizing the rate of formation of haloes of different mass at different times in hierarchical clustering models, as in the Press-Schechter approximation. The standard approach to this problem is to filter the density field in such models spatially with a spherical window function and to apply the top-hat model to predict the nonlinear collapse of initially linear-amplitude density fluctuations whose wavelength corresponds to the size of the window function. In a similar way, we might expect the TIS solution found here to be useful in characterizing the internal structure of the virialized objects which result from this nonlinear collapse. We note that there is currently no fundamental derivation or detailed theoretical explanation of the empirical NFW profile, so it is difficult to say just which initial conditions are necessary to produce it and how different these can be from that of the top-hat or of the spherical infall solution.

Some attempt to address this was made recently by Huss, Jain and Steinmetz (1998), who added different amounts of velocity dispersion to the initial conditions of a spherically symmetric density perturbation evolved by N-body simulation to

(C) 0000 RAS, MNRAS 000, 000-000 
see if the NFW profile would still fit the end result of its collapse. They found that the NFW was a decent fit for a range of initial velocity dispersions, but that it was possible to fit the simulation results better with a profile that was flatter at small radii than the NFW profile, as flat as $r^{-0.5}$. Syer and White (1998), on the other hand, have argued that the actual halo profiles in hierarchical clustering models are the product of the merging history of subclumps to form the halo, as an explanation for the cuspy inner density profile. They suggest that the slope of this inner density profile is different for haloes arising from linear density fluctuations whose initial power spectra have different slopes. If so, then the NFW is not a unique fit, since the actual profiles must depend upon the slope of the power spectrum at the wavelengths responsible for producing haloes of a given mass. This suggests that the haloes observed in N-body simulations are not fully relaxed, in the sense that they have not lost memory of the initial inhomogeneities which led to their formation. According to Syer and White (1998), in fact, the faster the initial density fluctuation power spectrum $P(k) \propto k^{n}$ decreases with increasing $k$, the shallower is the predicted central density profile of the haloes which form on this scale, with $\rho \propto r^{-\gamma}$, and $\gamma \approx 3(3+n) /(5+n)$. For the particular case of galactic scales in the standard CDM model, the effective power-law index is $n \approx-2.5$ and the scaling law would predict $\gamma \approx 0.6$, smaller than the NFW value. Only for larger-mass haloes, those at galaxy cluster scales, where $n \approx-2$, does this argument predict that $\gamma \approx 1$ as in the NFW profile. We note that, according to the scaling law of Syer and White (1998), as $n$ approaches -3 , the approximate asymptotic value in the small wavelength limit in the CDM model, the inner profile is predicted to flatten to a constant core value. It is tempting to speculate that the scaling argument, if correct, might be pointing to our TIS solution for the virialized inner portion of a halo which condenses out of a CDM model universe, for smaller mass haloes, those which originate from density fluctuations in the small wavelength limit. In this limit, there are mass fluctuations of comparable amplitude on all scales, collapsing out nearly simultaneously, which may be the limit in which the phenomenon of repeated mergers of isolated, dense subclumps to build up a larger halo is not prevalent enough to generate a central density cusp.

We have focused above on the differences between the NFW and TIS profile behaviour at small radii, but there is also a difference at large radii; the logarithmic slope of the TIS density profile drops as low as -2.5 at intermediate radii $\left(r / r_{0} \approx 9\right)$ and rises to -2.1 at the outer radius (see Fig.3), whereas the outer slope of the NFW profile approaches -3 (i.e. at the outer radius, $\left(r / r_{S}\right)_{\max }$, the NFW slope is $\left.3-2 /\left(\left(r / r_{S}\right)_{\max }+1\right)\right)$. This difference may reflect the transition between the inner virialized part of the halo, which we have characterized as isothermal and hydrostatic in formulating our TIS solution, and the outer region, involving continuous infall, which is neither isothermal, nor hydrostatic. The infall, itself, is generally predicted by spherical models to have a density profile closer to $r^{-2}$ than to $r^{-3}$ (e.g. Gunn and Gott 1972; Fillmore and Goldreich 1984; Hoffman and Shaham 1985; Bertschinger 1985; Moutarde, et al. 1995). When account is taken of the presence of an accretion shock in the fluid case or of density caustics and shell-crossing in the case of collisionless matter, such as the self-similar infall solution of Bertschinger (1985), the density profile inside the shocked region and the region of shell-crossing is also closer to $r^{-2}$ than to $r^{-3}$, even before any dynamical relaxation of the matter to an equilibrium profile occurs. Across the boundary between these two regions, the outer region of infalling matter and the inner region of shocked fluid and collisionless shell-crossing, however, there is a density jump, with a higher density inside the outermost density caustic than just outside this radius. To the extent that such a spherical model can represent the average behaviour of a more inhomogeneous initial condition, therefore, we expect the average density in the more general case to drop more steeply than $r^{-2}$ across some transition zone at $r \sim r_{\mathrm{vir}}$ where the actual transition in the collisionless matter is not discontinuous but is rather of finite radial extend. This would account qualitatively for the steepening in the NFW profile from $r^{-2}$ to $r^{-3}$ at large radii.

The question of whether the haloes in the N-body simulations of the CDM model, fit by the NFW profile, are relaxed and in equilibrium has been partially addressed by Tormen, Bouchet and White (1998) for the case of collisionless matter in an Einstein-de Sitter universe in which the density fluctuations are Gaussian random noise with a power spectrum which is a scale-free power-law of index $n=-1$. They report that the haloes, while not isothermal, are not very far from isothermal. They also find that the particle velocities at small radii are nearly isotropic while those at large radii are predominantly radial. As another approach, these authors compared different mass estimators for a set of haloes, all based upon an application of the Jeans equation for the dynamical equilibrium of a spherically symmetric collisionless system, allowing for different degrees of departure from the condition of velocity dispersion isothermality and isotropicity. They found that these mass estimators all compared favorably with the true mass if applied to estimate the total mass interior to the virial radius, while the estimator which took account of anisotropic velocity dispersion was in good agreement with the true mass at all radii. This suggests that the haloes are approximately and on average in a state of dynamical equilibrium but not fully relaxed to the point of having isotropic and isothermal velocity dispersion. As an additional probe, Tormen et al. (1997) also considered the fate of their simulated haloes at late times under the artificial circumstance in which a halo identified at some epoch is allowed to evolve further but with all matter removed outside of a sphere of radius twice the size of the original halo. This was intended to break the clustering hierarchy so as to allow the halo to evolve to a relaxed equilibrium state in the absence of further influx of perturbing substructure. Interestingly, the density profiles of the resulting haloes tended to flatten thereafter at very small radii, although the authors suggested that this might reflect some numerical error or artifact. Otherwise, the haloes showed less substructure with time but continued to be well fit, outside the central region, by the NFW profile.

What do numerical N-body simulations tell us about the accuracy or universality of the NFW profile for the haloes that

(c) 0000 RAS, MNRAS 000, 000-000 
form in the particular Gaussian random noise, hierarchical clustering model, CDM, which it was originally proposed to fit? Unfortunately, the results to date are not yet conclusive. For the purposes of discussion, it is useful to consider a broader family of density profiles, of the following form:

$\rho(r)=\frac{\rho_{0}}{\left(\frac{r}{r_{S}}\right)^{\gamma}\left(1+\left(\frac{r}{r_{S}}\right)^{1 / \alpha}\right)^{\alpha(\beta-\gamma)}}$

(e.g. Hernquist 1990; Zhao 1996). This density profile approaches a power law of slope $-\gamma$ at small radii, and a power law of slope $-\beta$ at large radii, with a break at the characteristic radius $r_{S}$ and a transition region between whose width increases with increasing $\alpha$. The NFW profile corresponds to the parameters $(\alpha, \beta, \gamma)=(1,3,1)$. An earlier suggestion of a galactic halo density profile for elliptical galaxies by Hernquist (1990) corresponds to $(\alpha, \beta, \gamma)=(1,4,1)$. A comprehensive review of the attempts to fit numerical simulation results for halo density profiles to those of the form in equation (106) above is beyond the scope of the present paper. We will mention only a few of the most recent and refer the reader to these papers and references therein for further description. Tormen et al. (1997) compare their simulation results by an N-body tree-code for haloes in the case of a scale-free power-law power spectrum of initial density fluctuations with $n=-1$ to the NFW and Hernquist profiles, resolving individual haloes with 20,000 simulation particles. Fitting the region between 0.01 and one times the virial radius (defined roughly in terms of the conventional SUS approximation for the average overdensity within a postcollapse, virialized top-hat), they find a somewhat better fit for the NFW profile than for the Hernquist profile. Recent high-resolution simulations of galaxy halo formation in the CDM model by Kravtsov, et al. (1998), however, by a new Adaptive Refinement Tree (ART) N-body code with a comparable number of particles per halo and a larger sample of haloes to analyze find a systematic deviation from the NFW profile at small radii, with a much flatter central profile, corresponding to $\rho(r) \propto r^{-\gamma}$ with $\gamma \approx 0.2$ (i.e. $(\alpha, \beta, \gamma) \approx(0.5,3,0.2)$; we note that Kravtsov, et al. define their own $\alpha$ as the inverse of the one we use here, so we transform their value to our notation). At small radii, at least, this is perhaps more consistent with the flat core of the TIS solution than with the cuspy center of the NFW density profile. On the other hand, very high resolution N-body simulations by a parallel N-body tree-code, reported by Moore, et al. (1998), of two rich-cluster-sized haloes in a CDM model in which a range of resolutions up to more than $10^{6}$ particles within the virial radius was used, finds that the simulated profiles deviate at small radii from NFW in the opposite sense, becoming as steep as $\rho(r) \propto r^{-\gamma}$ with $\gamma \approx 1.4($ i.e. $(\alpha, \beta, \gamma) \approx(0.7,2.8,1.4)$ ), which they believe is reliably determined down to radii less than 0.01 times the virial radius. A qualitatively similar result regarding the density cusp is reported by Fukushige and Makino (1998) based upon their N-body simulations of the formation of a single, massive, galactic halo in the CDM model by direct summation of two-body gravitational forces utilizing the Grape-4 special-purpose computer. The full simulation volume of comoving radius $2 \mathrm{Mpc}$ in present units contained 786,000 particles and more than $10^{12} M_{\odot}$, but they do not indicate what fraction of these particles were contained within the virial radius of the final object.

There are at least two possible explanations for the different results of these latest simulations. It may be that, as Syer and White (1998) suggest, the NFW profile is not universal but depends instead on the shape of the density fluctuation power spectrum at wavelengths responsible for the particular haloes in question. As such, the galactic halo profiles of Kravtsov, et al. are expected to be flatter at small radii than NFW, as described above, in contrast to the large, cluster-mass haloes of Moore, et al. (1998). This scaling argument alone does not, however, explain why the Moore et al. (1998) haloes are more cuspy even than those of Tormen et al. (1997) for the scale-free $n=-1$ case, since, although the effective $n$ in the CDM model does increase from galactic to cluster mass scales, it does not exceed -1 on the cluster scale, so the Tormen et al. (1997) results, too, should show a greater degree of central cuspiness than does NFW and they do not. Nor does this explain why the simulations of Fukushige and Makino (1998) of a galactic halo, rather than of a cluster halo, also show a density cusp steeper than the NFW profile. A second possibility is that, despite the improvement in mass resolution afforded by these new simulations, numerical effects are still responsible for a failure to resolve the inner regions of the haloes properly. Splinter et al. (1998) for example, have raised a significant question about whether artificial collisional relaxation effects in N-body codes used to model collisionless matter have been underestimated, suggesting that previous claims of length resolution below the mean interparticle separation are not correct. Further work will be necessary to resolve this matter.

Regarding the question of the possible difference between the gas and collisionless dark matter distributions, we have assumed this difference to be relatively small in our TIS derivation, so that our TIS solution can be applied to either component, in proportion to its relative share of the total mean matter density in the unperturbed background universe. This was motivated, in part, by the self-similar spherical infall solution of Bertschinger(1985) which supports this assumption if the gas has a ratio of specific heats $\gamma=5 / 3$. It is also supported by coupled N-body and gas dynamical simulations of the CDM model (i.e. those which neglect both radiative cooling and external input of energy to the gas, such as by photoionization and supernova explosions). For example, cluster simulations by Eke, Navarro, and Frenk (1998) find that the cumulative gas mass fraction measured from the cluster center (in units of the universal mean value over all space) is unity outside the virial radius, roughly 0.9 between $0.5 r_{\text {vir }}$ and $r_{\text {vir }}$ and drops below this significantly only in the core. Interestingly, while the density profile of the cluster dark matter in those simulations is well-fit by the NFW profile, the gas density profile does depart somewhat in the center, where it is found to possess a finite core, and it is better-fit overall by a $\beta$-model like that traditionally used to

(c) 0000 RAS, MNRAS 000, 000-000 
model cluster X-ray gas as a hydrostatic isothermal sphere, with $\beta$ between 0.7 and 0.75 for the average profiles at different epochs, closer to our TIS solution than to the NFW profile of the dark matter. The ratio of the virial radius to the core radius of the average cluster density profile at $z=0$, in fact, was found to be 20 , as compared to our TIS solution in which the ratio of truncation radius to core radius is 29.4. (Note: our truncation radius is, moreover, a little higher than the SUS approximation for $r_{\mathrm{vir}}$, so if we replace $r_{t}$ by $r_{\mathrm{vir}}=0.5 r_{m}$, our ratio drops to 26, even closer). Bryan and Norman(1998) report that their simulations of cluster formation in the CDM model by a different numerical technique also support the assumption that the cluster gas density profile follows the dark matter density profile throughout most of the cluster volume, with differences important only at $r / r_{\text {vir }}<0.04$. In short, our approximation that the same density profile can be used to describe both gas and dark matter in the postcollapse virial equilibrium of a top-hat density perturbation seems justified, in view of these simulation results and the uncertainty described above in determining the true nature of the dark matter profiles at small radii (i.e. radii as small or smaller than the core radius of our TIS solution).

We note that, while the dark matter in these simulated haloes in the CDM model is generally found to exhibit velocity dispersion which is somewhat radially-biased outside the core, the bias is relatively modest except at radii close to $r_{\text {vir }}$. In particular, if $\beta_{a n} \equiv 1-\bar{v}_{t}^{2} / 2 \bar{v}_{r}^{2}$, where $v_{t}\left(v_{r}\right)$ correspond to tangential (radial) velocities, then Eke et al. (1998) report $\beta_{a n}<0.2$ out to $r=r_{\text {vir }} / 2$, rising only to 0.4 at $r \approx r_{\text {vir. }}$. The gas, of course, cannot have an anisotropic temperature but could share the radial bias in the sense of there being a net infall or outflow, if it were not hydrostatic. The radial velocity bias of the dark matter in the outskirts of the cluster need not be inconsistent with our assumption that the gas follows the dark matter as long as it reflects the transition from infall at larger radii to hydrostatic equilibrium at smaller radii for both the gas and the dark matter, as already described above to explain the steeper $r^{-3}$ dependence of the NFW profile at these radii, compared to $r^{-2}$ for the TIS solution. The ratio of dark matter velocity dispersion to gas temperature is reported to be close to unity throughout the simulated clusters, except at the very center, where the gas remains isothermal but the dark matter "temperature" drops, inside the gas density core radius, in the inner region where the cuspy density of dark matter is claimed to occur. In short, there are many indications from these simulations that our TIS solution is a plausible approximation for the behaviour of the gas, and also some indication from the similarity between the gas and dark matter outside the gas density core that our assumption that gas and dark matter follow the same solution is valid.

Finally, we return to the question of whether the NFW profile for the dark matter in haloes that form in the CDM model is in agreement with the observed properties of dark matter haloes in nature. A full treatment of this question is outside the scope of our discussion. We focus, instead on a few recent attempts to answer this question on two widely different scales. The first involves dwarf and low-surface-brightness (LSB) late-type galaxies. Such systems are believed to be dark-matter dominated, and observations of their rotation curves have been used to probe the density structure of dark matter haloes directly. Flores and Primack(1994) and Moore(1994) used rotation curve measurements for several such galaxies and concluded that the central density distribution could not be as cuspy as the $r^{-1}$ shape of a NFW profile. Burkert(1995) showed that the data of Moore(1994) was well fit by a profile of the form

$\rho(r)=\frac{\rho_{0}}{\left(1+\frac{r}{r_{S}}\right)\left[1+\left(\frac{r}{r_{S}}\right)^{2}\right]}$,

which differs significantly from the NFW profile in that it exhibits a finite core. Kravtsov et al.(1998) have examined this question with a somewhat larger observed sample of dwarf and LSB galaxy rotation curves and concluded that a good fit to these halo density profiles of the form in equation (106) involves a shallow central profile $\rho \propto r^{-\gamma}$, with $\gamma=0.2-0.4$, shallower than the NFW profile. Burkert and Silk(1997) reexamined the rotation curve of one dwarf galaxy, DDO154, to suggest that the discrepancy with the NFW profile could be resolved if a spheroid of dark baryons were added to the CDM halo of comparable mass. In short, on galaxy scales, the well-studied systems which are considered to be the most direct measure of the dark matter halo are found to be inconsistent with the NFW in requiring either a finite core or a shallow inner profile. This is the same sense as the discrepancy between the TIS and NFW profiles.

On the larger scale of clusters of galaxies, the observations of the X-ray emission by the gas and of gravitational lensing by the dark matter provide two independent measures of the distribution of the dark matter in the cluster. Makino, Sasaki and Suto 1998) found that the gas density profile of an X-ray cluster, if modeled as an isothermal sphere in hydrostatic equilibrium with a dark matter potential well which follows the NFW density distribution, yields an isothermal $\beta$-profile with a finite core, as required to match the observations of X-ray cluster brightness profiles, but with too small a core radius relative to the observed ones. Eke et al.(1998) performed SPH simulations of the formation of such clusters in a CDM model and claim that their cluster dark matter follows the NFW profile while the gas density profiles are well fit by the isothermal $\beta$-model, with a typical core radius for the latter of about $100 h^{-1} \mathrm{kpc}$ at $z=0$, in a good agreement with observations. The origin of the difference between these two conclusions is not clear.

An independent comparison of the dark matter density profiles in observed clusters of galaxies and the NFW profile is provided by a recent observation of strong gravitational lensing of a background galaxy by the galaxy cluster CL $0024+1654$ at $z=0.39$ by Tyson, Kochanski, and Dell'Antonio(1998). These authors construct a high-resolution map of the central mass distribution of the cluster and find it to be inconsistent with the singular density profile of NFW. The observed cluster requires 
a smooth dark matter distribution with a $35 h^{-1} \mathrm{kpc}$ core and a slope that is slightly shallower than that of an isothermal sphere. This is consistent with the TIS solution presented here.

\subsection{Comparison of the predicted virial temperature and mass - radius - temperature scaling laws of the minimum-energy TIS solution with numerical simulation results and observations of X-ray clusters}

We have noted above that our minimum-energy TIS solution temperature is larger by a factor of $b_{T}=2.16$ than the postcollapse virial temperature calculated in the SUS approximation, while it is smaller by a factor of $3 / 2.16=1.39$ than the virial temperature in the limit of a singular isothermal sphere. How does the TIS temperature compare with the results of numerical simulations of cosmological structure formation? The clearest comparison available to date is with the results of numerical gas dynamics simulations of X-ray cluster formation in the CDM model. It is customary to describe these results by comparing the numerical gas temperatures with the so-called "virial temperature" $T_{\text {vir }}$ of the clusters. Unfortunately, different authors define their $T_{\text {vir }}$ differently, often without explicit justification, which makes a direct comparison more difficult. We shall attempt to compare the results quoted in the literature uniformly by referring to the definition of virial temperature used by Kitayama and Suto(1997), given by

$k_{B} T_{\text {vir }} \equiv \gamma_{\text {vir }} \frac{G \mu m_{p} M}{3 r_{\text {vir }}}$

in which $r_{\mathrm{vir}}$ is the virial radius, which different authors have chosen to define in different ways when analyzing their simulations (e.g. sometimes $r_{\text {vir }}$ is set equal to $r_{200}$, the radius within which the average density is 200 times the mean background value), and $\gamma_{\mathrm{vir}}$ is a "fudge factor" which typically ranges between $\gamma_{\mathrm{vir}} \approx 1$ and $\gamma_{\mathrm{vir}} \approx 1.5$ and is chosen either to match simulation results or according to some analytical model like the SUS approximation. Kitayama and Suto(1997), for example, adopted the value $\gamma_{\text {vir }}=1.2$ based upon the gas dynamical simulations of White et al. (1993), while pointing out that other authors adopted other values in their analyses, including $\gamma_{\text {vir }}=1$ (Kitayama \& Suto 1996), $\gamma_{\text {vir }}=1.1$ (Viana and Liddle 1996), and $\gamma_{\text {vir }}=1.5$ (Eke et al. 1996). A complete review of which authors adopt which value of $\gamma_{\text {vir }}$ in defining $T_{\text {vir }}$ is outside the scope of this paper. We shall focus, instead, on some recent papers for which simulation results can be used to determine the value of $\gamma_{\text {vir. }}$.

We note that the same expression used in equation (108) can be used to define the virial velocity dispersion, $\sigma^{2}$, of the collisionless dark matter component, according to equation (11), if we replace $k_{B} T_{\text {vir }} /\left(\mu m_{p}\right)$ in equation (108) by $\sigma^{2}$. Henceforth we shall distinguish the $\gamma_{\text {vir }}$-values for the gas and the dark matter by the subscripts " $T$ " for the gas and " $\sigma$ " for the dark matter, and drop the subscript "vir" for simplicity. Our assumption in this paper is that the gas and dark matter components each follow the same TIS profile with component densities which are simply proportional to the total density at each point, and that the two components have the same virial temperature or equivalent virial velocity dispersion $\sigma^{2}$. The latter property is sometimes quantified by defining a parameter $\beta_{\sigma-T} \equiv \mu m_{p} \sigma^{2} / k_{B} T$ (i.e. $\beta_{\sigma-T}=\gamma_{\sigma} / \gamma_{T}$ ). We have assumed here that $\beta_{\sigma-T}=1$ is a good approximation.

A comparison of our TIS solution result, the SUS and SIS approximations, and the results of some of the more recent numerical simulation studies of cluster formation in the CDM model is presented in Table 2. For the TIS solution evaluated at the boundary $\zeta=\zeta_{t}, \gamma_{T}=\gamma_{\sigma}=(6 / 5)\left(b_{T} \eta\right)=1.43$ and $\beta_{\sigma-T}=1$, while the SUS approximation corresponds to $\gamma_{T}=\gamma_{\sigma}=0.6$ and $\beta_{\sigma-T}=1$, and the SIS approximation yields $\gamma_{T}=\gamma_{\sigma}=1.5$ and $\beta_{\sigma-T}=1$. The numerical gas dynamical simulations all report $\beta_{\sigma-T} \approx 1$, with $\gamma_{T}$ and $\gamma_{\sigma}$ ranging between 1.2 and 1.5. The purely collisionless $N$-body simulations (without gas dynamics) also find $\gamma_{\sigma}$ between 1.2 and 1.5. These results are in good agreement with the prediction of our TIS solution, in rough agreement with the SIS approximation and in clear disagreement with the SUS approximation. At a more detailed level, there are some disagreements between the TIS solution and the simulation results and amongst the simulation results themselves. For example, Eke et al. (1998) report that the region of hydrostatic and virial equilibrium is most reliably located at $r \simeq r_{\text {vir }} / 3$ and Evrard et.al. (1996) (hereafter EMN96) note a similar trend, although departures from equilibrium are never very great even at $r_{\text {vir }}$. Similarly, while the clusters are found in all cases to be approximately isothermal, there is some tendency for the temperature to fall by less than a factor of two roughly between $r_{\mathrm{vir}} / 2$ and $r_{\mathrm{vir}}$. Bryan and Norman(1998) find a small tendency for $\beta_{\sigma-T}$ to decrease with cluster mass, smaller than unity for smaller clusters, but approaching unity for the larger clusters, which they attribute to the effect of numerical resolution in better resolving the more massive clusters. Finally, Eke et al. (1998) find a small drop in the dark matter $\sigma^{2}$ relative to the gas temperature $T$ near the center, consistent with their finding that the central density of the dark matter is more centrally peaked than is that of the gas. Some of the differences amongst the simulation results may reflect a weak dependence of those results on the different background cosmology models adopted. Nevertheless, within the uncertainties of the simulation results, there is good global agreement between the TIS solution presented here and the numerical simulations of cluster formation in the CDM model in terms of the temperature - mass - radius virial relation.

EMN96 have used a large set of simulated clusters to infer a mass-temperature scaling law and a related radius-temperature scaling law for the clusters. While the functional dependences of mass and radius on temperature in these scaling laws are

(C) 0000 RAS, MNRAS 000, 000-000 
Table 2. Comparison of the minimum-energy TIS solution and X-ray cluster simulation results for the mass-temperature virial relation

\begin{tabular}{|c|c|c|c|}
\hline Source & $\gamma_{T}$ & $\gamma_{\sigma}$ & $\beta_{\sigma-T}$ \\
\hline \multicolumn{4}{|l|}{ Simulation Results } \\
\hline Eke, Navarro, and Frenk (1998)........... & 1.5 & 1.5 & 1 \\
\hline Bryan and Norman (1998)..................... & 1.2 & 1.2 & 1 \\
\hline Evrard, Metzler, and Navarro (1996)... & 1.4 & 1.4 & 1 \\
\hline Crone and Geller (1995)........................ & - & 1.2 & - \\
\hline 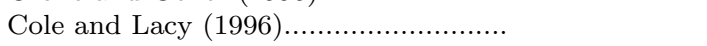 & - & $\approx 1.5$ & - \\
\hline \multicolumn{4}{|l|}{ Analytical Results } \\
\hline 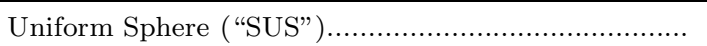 & 0.6 & 0.6 & 1 \\
\hline Singular Isothermal Sphere ("SIS") ........................... & 1.5 & 1.5 & 1 \\
\hline Truncated Isothermal Sphere ("TIS", this paper) $a$... & 1.43 & 1.43 & 1 \\
\hline
\end{tabular}

${ }^{a}$ For the TIS solution, $\gamma_{\text {vir }}$ is evaluated at the outer boundary, $\zeta=\zeta_{t}$. The values for other radii are plotted in Figure 6, showing that $\gamma_{\mathrm{vir}}$ varies roughly between 1.2 and 1.5 for radii between 0 and $r_{t}$.

consistent with the crude expectations of virial equilibrium as measured at a fixed value of the average density contrast inferior

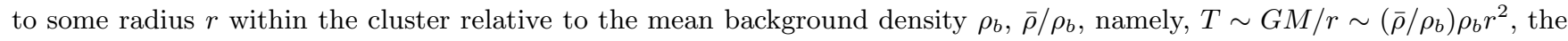
coefficients of the scaling laws are determined empirically from the numerical gas dynamical simulations of X-ray cluster formation in the CDM model. By making a conservative choice, $\rho / \rho_{b}=500$, of an average density contrast which is generally expected to occur at a radius which is significantly smaller than that within which the cluster is well-approximated by hydrostatic and virial equilibrium, EMN96 are able to calibrate these scaling laws numerically, with relatively small scatter, with the intention of comparing them with X-ray observations of clusters to determine reliable mass estimates for the clusters. For our purposes, these numerically - calibrated scaling laws provide an excellent check on our analytical TIS model. If our TIS model solution proves to be in good agreement with these scaling laws, then we will not only have confirmed our TIS analytical predictions but also have found in our TIS solution a derivation of these numerically - calibrated scaling laws.

Let $r_{500}$ be the radius of the sphere within which the mean density contrast is $\bar{\rho} / \rho_{b}=500$, and let $M_{500}$ be the mass enclosed by this sphere. EMN96 find

$$
\begin{aligned}
M_{500}(T) & =(1.11 \pm 0.16) \times 10^{15}\left(\frac{T}{10 \mathrm{keV}}\right)^{3 / 2} h^{-1} M_{\odot}, \\
r_{500}(T) & =(1.24 \pm 0.09)\left(\frac{T}{10 \mathrm{keV}}\right)^{1 / 2} h^{-1} \mathrm{Mpc}
\end{aligned}
$$

where $T$ is an appropriate average temperature for the cluster gas. The simulation results which led to equations (109) and (110) were analyzed at $z=0.04$, but scaled to $z=0$ assuming that they both scale as $(1+z)^{-3 / 2}$, so there is no information explicitly contained in these equations regarding the scaling with redshift. The corresponding scaling laws for a mean density contrast $\bar{\rho} / \rho_{b}=200$, were presented in Arnaud and Evrard 1998) (with no error bars specified, but the error bars should be larger than those at smaller radii; Evrard, private communication) for the same numerical simulation results as in EMN96, according to:

$$
\begin{aligned}
M_{200}(T) & =1.45 \times 10^{15}\left(\frac{T}{10 \mathrm{keV}}\right)^{3 / 2} h^{-1} M_{\odot}, \\
r_{200}(T) & =1.85\left(\frac{T}{10 \mathrm{keV}}\right)^{1 / 2} h^{-1} \mathrm{Mpc} .
\end{aligned}
$$

We can derive the corresponding relations according to our minimum-energy TIS solution. Let $X$ be the average density contrast inside a sphere of radius $r_{X}$ which encompasses a total mass $M_{X}$. Then the minimum-energy TIS solution derived here can be used to solve for $M_{X}$ and $r_{X}$ for the postcollapse virialized object of virial temperature $T$ which results from a top-hat which collapses at any $z_{\text {coll }}$, for any overdensity $X$, as follows. Let $\bar{\rho}_{X}$ be the average density inside this sphere of radius $r_{X}$. In terms of $\rho_{b}\left(t_{\mathrm{coll}}\right)$, the mean cosmic background density at the time $t_{\text {coll }}$ for the top-hat perturbation which created the postcollapse virialized object described by the minimum-energy TIS solution, the density contrast is $X=\bar{\rho}_{X} / \rho_{b}\left(t_{\text {coll }}\right)$. We shall continue to refer to the values of these quantities at the outer radius $r_{t}$ by using the subscript "t" as we have in previous sections. In terms of the dimensionless radius $\zeta$ and mass $\tilde{M}$, the fact that $\bar{\rho}_{X} \propto M_{X} / r_{X}^{3}$ allows us to write

$\frac{\tilde{M}_{X} / \zeta_{X}^{3}}{\tilde{M}_{t} / \zeta_{t}^{3}}=\frac{X}{\bar{\rho}_{t} / \rho_{b}\left(t_{\text {coll }}\right)}$. 


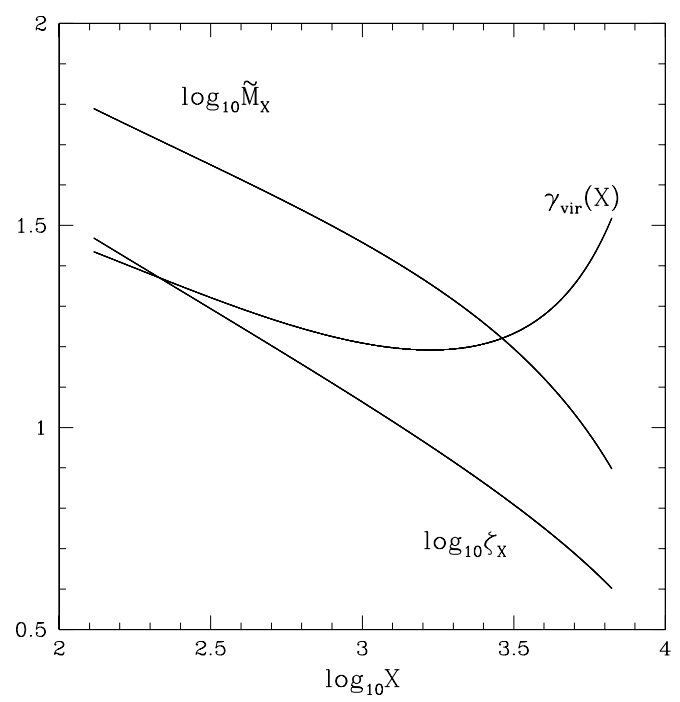

Figure 6. The dimensionless radius $\zeta_{X}$, and the corresponding mass $\tilde{M}_{X}$, within which the average density with respect to the background is $X$, and the factor $\gamma_{\mathrm{vir}}$, as functions of the density $X$ for our minimum-energy TIS solution.

Equations (102) and (113) combine to yield an implicit equation for $\zeta_{X}$ and, hence, for $\tilde{M}_{X}$, which can be solved numerically for any given $X$, according to

$\frac{X \tilde{\rho}_{X}^{3}}{\tilde{M}_{X}}=18 \pi^{2}\left(\frac{b_{T}}{5}\right)^{3} \tilde{M}_{t}^{2}$.

The mass $M_{X}$ can be expressed in terms of the resulting $\tilde{M}_{X}$, using equations (34), (66), and (67), according to

$\frac{M_{X}}{\tilde{M}_{X}}=\frac{1}{3 \pi G}\left(\frac{b_{T}}{5}\right)^{-3 / 2} \tilde{M}_{t}^{-1}\left(1+z_{\mathrm{coll}}\right)^{-3 / 2} H_{0}^{-1}\left(\frac{k_{B} T}{\mu m_{p}}\right)^{3 / 2}$.

Similarly, the radius $r_{X}$ can be expressed in terms of the dimensionless radius $\zeta_{X}$ which is the solution of equation (114. and the core radius $r_{0}$, according to

$\frac{r_{X}}{\zeta_{X}}=r_{0}=G \frac{\mu m_{p}}{k_{B} T} \frac{M_{X}}{\tilde{M}_{X}}=\frac{1}{3 \pi}\left(\frac{b_{T}}{5}\right)^{-3 / 2} \tilde{M}_{t}^{-1}\left(1+z_{\mathrm{coll}}\right)^{-3 / 2} H_{0}^{-1}\left(\frac{k_{B} T}{\mu m_{p}}\right)^{1 / 2}$,

where in expressing $r_{0}$ we used equations (28) and (34). Equations (114), (115), and (116) constitute the final scaling laws for the mass and radius within our TIS for the interior spherical region of average density contrast $X$, in terms of the predicted virial temperature $T$, collapse epoch $z_{\text {coll }}$ and Hubble constant $H_{0}$, if we use the results of our TIS solution to evaluate $b_{T}$ and $\tilde{M}_{t}$. Plots of $\zeta_{X}$ and $\tilde{M}_{X}$ as functions of $X$, obtained numerically from the TIS solution, are shown on Fig. A, along with a plot of $\gamma_{\text {vir }}(X) \equiv 3 \tilde{M}_{X} / \zeta_{X}$ which enters equation (108) if we wish to evaluate equation (108) using any radius $r \leq r_{t}$ and replace $M$ by $M(\leq r)$ and $r_{\text {vir }}$ by $r$.

In order to compare directly with the empirical scaling laws of EMN96 in equations (109), (110), (111), and (112) above, we set $X=500$ or $X=200$, accordingly, and use our minimum-energy TIS solution summarized in Table 1 to evaluate equations (114) $-(116)$. We find $\zeta_{500}=15.96, \tilde{M}_{500}=37.74, \zeta_{200}=24.20$, and $\tilde{M}_{200}=52.65$. The analytical TIS scaling laws which result are the following

$$
\begin{aligned}
M_{500}(T) & =1.11 \times 10^{15}\left(\frac{T}{10 \mathrm{keV}}\right)^{3 / 2}\left(1+z_{\mathrm{coll}}\right)^{-3 / 2}\left(\frac{\mu}{0.59}\right)^{-3 / 2} h^{-1} M_{\odot}, \\
r_{500}(T) & =1.24\left(\frac{T}{10 \mathrm{keV}}\right)^{1 / 2}\left(1+z_{\mathrm{coll}}\right)^{-3 / 2}\left(\frac{\mu}{0.59}\right)^{-1 / 2} h^{-1} \mathrm{Mpc}
\end{aligned}
$$

and

$$
\begin{aligned}
M_{200}(T) & =1.55 \times 10^{15}\left(\frac{T}{10 \mathrm{keV}}\right)^{3 / 2}\left(1+z_{\text {coll }}\right)^{-3 / 2}\left(\frac{\mu}{0.59}\right)^{-3 / 2} h^{-1} M_{\odot}, \\
r_{200}(T) & =1.88\left(\frac{T}{10 \mathrm{keV}}\right)^{1 / 2}\left(1+z_{\text {coll }}\right)^{-3 / 2}\left(\frac{\mu}{0.59}\right)^{-1 / 2} h^{-1} \mathrm{Mpc} .
\end{aligned}
$$


Table 3. Comparison of the minimum-energy TIS solution and X-ray cluster simulation results for the radius-temperature relation.

\begin{tabular}{llllll}
$X=\bar{\rho} / \rho_{b}$ & $\zeta_{X}$ & $\tilde{M}_{X}$ & {$\left[r_{10}(X)\right]_{E M N 96}$} & {$\left[r_{10}(X)\right]_{T I S}$} & $($ TIS -EMN96)/EMN96 \\
\hline $200 \ldots \ldots$ & 24.20 & 52.65 & 3.7 & 3.759 & 0.016 \\
$250 \ldots$. & 21.88 & 48.59 & 3.37 & 3.398 & 0.008 \\
$500 \ldots$. & 15.96 & 37.74 & 2.48 & 2.479 & 0.0006 \\
$1000 \ldots$ & 11.56 & 28.69 & 1.79 & 1.796 & 0.003 \\
$2500 \ldots$ & 7.32 & 18.21 & 1.11 & 1.137 & 0.024 \\
\hline
\end{tabular}

a Simulation results are from EMN96, Table 5, p. 505, with the value for $X=200$ as quoted in Arnaud and Evrard (1998).

Adopting the fully ionized gas value $\mu=0.59$ and the value of $z_{\text {coll }}=0$ appropriate for a direct comparison with the simulation results of EMN96, equations (117), (118), (119), and (120) above become

$$
\begin{aligned}
M_{500}(T) & =1.11 \times 10^{15}\left(\frac{T}{10 \mathrm{keV}}\right)^{3 / 2} h^{-1} M_{\odot}, \\
r_{500}(T) & =1.24\left(\frac{T}{10 \mathrm{keV}}\right)^{1 / 2} h^{-1} \mathrm{Mpc} .
\end{aligned}
$$

and

$$
\begin{aligned}
M_{200}(T) & =1.55 \times 10^{15}\left(\frac{T}{10 \mathrm{keV}}\right)^{3 / 2} h^{-1} M_{\odot}, \\
r_{200}(T) & =1.88\left(\frac{T}{10 \mathrm{keV}}\right)^{1 / 2} h^{-1} \mathrm{Mpc} .
\end{aligned}
$$

These are in remarkably close agreement with the empirical relations of EMN96 in equations (109), (110), (111), and (112), differing from them in radius by less than $2 \%$ and in mass by less than $7 \%$, well within the error bars of those empirical relations, an excellent check on our TIS solution. In that respect, our TIS solution, based upon the simplifying assumptions of spherical symmetry, isothermality and hydrostatic equilibrium, provides an approximate analytical derivation of the numerically calibrated scaling laws! This close agreement between the predictions of our analytical TIS solution and the numerical simulation results which arise as the dynamical outcome of more complicated initial conditions suggests that our simplifying assumptions are reasonable.

A further comparison with the numerical simulation results of EMN96 for the cluster gas is possible in which we compare the coefficients of the radius - temperature relation at different radii, corresponding to a range of density contrasts $X$, derived by us according to equation (114) above, with the numerical values reported in Table 5 of EMN96. EMN96 fit the simulation results for $z=0.04$ (divided by $(1+z)^{-3 / 2}$ to yield the scaling law at $z=0$ ) and $h=0.5$ to a relation of the form

$r_{X}(T)=r_{10}(X)\left(\frac{T}{10 \mathrm{keV}}\right)^{1 / 2}$

A comparison of the analytical TIS predictions for $r_{10}(X)$ in this case, with the tabulated values of EMN96 at $X=$ $200,250,500,1000$, and 2500 is shown in Table 3 . The TIS and simulation values are once again in remarkably close agreement, the same to within 2.5 per cent at all radii, spanning over an order of magnitude of density contrast, including the canonical value of $X=200$ so widely used when cosmological simulations are analyzed in comparison with observations of $\mathrm{X}$-ray clusters for a wide range of purposes.

Recently, Hjorth, Oukbir, and van Kampen (1998) have provided a direct comparison between observations of X-ray clusters and the theoretical mass-temperature virial relation above, which EMN96 report as a good fit to simulation numerical results and which we have derived from our TIS solution. Hjorth et al. (1998) calibrate this relation using observations of eight clusters in which the temperatures $T$ are determined from recent X-ray data from the ASCA satellite while the cluster masses are determined independently by gravitational lensing measurements. They find that the EMN96 mass-temperature relation is in good agreement with their observationally-calibrated relation (i.e. within the observational uncertainties of roughly few $\times 10 \%)$. In that sense, we can also claim good agreement between our analytical TIS model mass-temperature relation and these observations.

Regarding the numerically-calibrated radius-temperature relation of EMN96 in equation (110), which we have reproduced exactly here by our analytically derived TIS solution, Mohr and Evrard (1997) have shown that observations of nearby X-ray clusters support this relation. Once again, the analytical prediction of the radius-temperature relation by the TIS solution presented here is, therefore, consistent with the observations of X-ray clusters. 
In short, for all its simplicity, the TIS solution derived here appears to predict the virial temperature and the integrated mass distribution of the X-ray clusters formed in the CDM model, according to detailed, 3D, numerical simulations of the latter, remarkably well. It also matches the mass-temperature and radius-temperature virial relations observed for nearby X-ray clusters. We are encouraged by this to think that the minimum energy TIS solution presented here will be quite useful as a tool in semi-analytical models of galaxy and cluster formation and as a guide for understanding both the detailed numerical simulation results and observations. Further study of the dynamical origin and stability of this equilibrium solution and of the sensitivity of the relaxation process which leads to this equilibrium state to initial conditions is warranted. In addition, it may be useful to generalize the results presented here to other cosmological background models, such as an open, matter-dominated model, or a flat model with a nonzero cosmological constant, in order to describe objects that form at late enough times in such models that departures from the Einstein - de Sitter model may arise. We will address this issue in a future paper. We expect that, for an open, matter dominated model, the structure of the postcollapse equilibrium object will be the same as that derived here for the Einstein - de Sitter case, when expressed in dimensionless units and when referred to the quantities at the maximum expansion epoch of the parent tophat. In other words, the density profile will still be as plotted in Figure 3, where the core and truncation radii are expressed in units of $r_{m}$ and the density is in units of $\rho_{S U S}$. In that case, since the parent top-hat in an open, matter-dominated background universe evolves at a different rate relative to the background universe as compared to one in an Einstein-de Sitter model, $r_{m}$ and $\rho_{S U S}$ will be different.

\section{ACKNOWLEDGMENTS}

This research was supported in part by NASA grants NAG5-2785, NAG5-7363, and NAG5-7821 and NSF grant ASC 9504046. PRS is grateful to his hosts at The Institute of Astronomy, UNAM, Mexico City, for their hospitality during an early stage of this work and for the 1997 National Chair of Excellence at UNAM which he received from Mexico's CONACyT. In addition, we are grateful for the hospitality of the Aspen Center for Physics and its Summer Astrophysics Workshop in June 1998 during the completion of this work. We thank our referee Vincent Eke for a careful reading of the paper and his helpful comments.

\section{REFERENCES}

Antonov V.A. 1962, Vestn. Leningrad. Gos. Univ. 7, 135. [English translation in: Dynamics of Globular Clusters, IAU Symp. 113, eds. J. Goodman and P. Hut, 1985 Reidel, Dordrecht.]

Arnaud M. and Evrard A.E. 1998, MNRAS, submitted astro-ph/9806353

Bertschinger E. 1985, ApJS, 58, 39

Binney, J. and Tremaine, S., 1987, Galactic Dynamics. Princeton University Press, Princeton

Bond J.R., and Myers S.T. 1996, ApJS, 103, 1

Bonnor W.B. 1956, MNRAS, 116, 351

Bryan G.L., and Norman M.L. 1998, ApJ, 495, 80

Burkert A. 1995, ApJ, 447, L25

Burkert A., and Silk J. 1997, ApJ, 488, 55

Carlberg R.G., and Couchman H.M.P. 1989, ApJ, 340, 47

Cavaliere A. and Fusco-Femiano R.1976, A\&A, 49, 137

Cen R. 1998, preprint astro-ph/9806074)

Chiése J.-P., Teyssier R., and Alimi J.-M. 1997, ApJ, 484, 40

Cole S. and Lacey C 1996, MNRAS, 281, 716

Crone M.M., and Geller M.J. 1995, ApJ, 110, 21

David L.P., Jones C., and Forman W. 1995, ApJ, 445, 578

Ebert R. 1955, Z. Astrophys., 37, 216

Ebert R. 1957, Z. Astrophys., 42, 263

Efstathiou G., and Rees M.G. 1988, MNRAS, 230, 5

Eke V.R., Navarro J.F., and Frenk C.S. 1998, ApJ 503, 569

Eke V.R., Cole S., and Frenk C.S. 1997, MNRAS, 282, 263

Evrard A.E., Metzler C.A., and Navarro J.F. 1996, ApJ, 469, 494 (EMN96)

Fillmore J.A. and Goldreich P. 1984, ApJ 281, 1

Flores R., and Primack J. R. 1994, ApJ, 427, L1

Freeman K.C. 1992, in Physics of Nearby Galaxies: Nature or Nurture?, (XIIth Moriond Astrophysics Meeting), eds. T. Xuan Thuan, C. Balkowski, and J. Tran Tranh Van. Editions Frontiéres, Paris, pp. 201-214

Fukushige T., and Makino J. 1998, ApJ, 477, L9

Gunn J.E., and Gott J.R. 1972, ApJ, 176, 1

Hairer E., Norsett S.P., and Wanner, G., 1996, Solving Ordinary Differential Equations, V.2 - Stiff and Differential-Algebraic Problems (2nd rev. ed.) Springer-Verlag, Berlin; New York

Hernquist L. 1990, ApJ, 356, 359

Hjorth J., Oukbir J., and van Kampen E. 1998, MNRAS, 298, L1

Hoffman Y., and Shaham J. 1985, ApJ, 297, 16 
Huss A., Jain B., and Steinmetz M. 1998, preprint astro-ph/9803117

Jones C., and Forman W., 1992, in Clusters and Superclusters of Galaxies, ed. A. C. Fabian Kluwer Academic Publishers, pp 49-70 Katz J. 1978, MNRAS, 183, 765

King I. R. 1966, AJ, 71, 64

King I. R. 1972, ApJ, 174, L123

Kitayama T. and Suto Y. 1996, MNRAS, 280, 638.

Kitayama T. and Suto Y. 1997, ApJ, 490, 557

Klypin A., Borgani S., Holtzman J. and Primack J. 1995, ApJ, 444, 1

Kravtsov A. V., Klypin A. A., Bullock J. S., and Primack J. R. 1998, ApJ, 502, 48

Lynden-Bell D. and Wood R. 1968, MNRAS, 138, 495

Makino N., Sasaki S., and Suto Y. 1998, ApJ, 497, 555

McCrea W.H. 1957, MNRAS, 117, 562

Mohr J.J., and Evrard A.E. 1997, ApJ, 491, 38

Monaco P. 1997, astro-ph/9710085

Moore B. 1994, Nature, 370, 629

Moore B., Governato F., Quinn T., Stadel J., and Lake G. 1998, ApJ, 499, L5

Moutarde F., Alimi J.-M., Bouchet F., and Pellat R. 1995, ApJ, 441, 10

Natarajan P. and Lynden-Bell D. 1997, MNRAS, 286, 268 (NL97)

Navarro J.F., Frenk C.S., and White S.D.M. 1996, ApJ, 462, 563

Navarro J.F., Frenk C.S., and White S.D.M. 1997, ApJ, 490, 493

Padmanabhan T. 1989, ApJS, 71, 651

Padmanabhan T. 1990, Phys. Reports, 188, No. 5, 285

Padmanabhan T. 1993, Structure Formation in the Universe Cambridge University Press, Cambridge

Peebles P.J.E., 1980, The Large Scale Structure of the Universe Princeton University Press, Princeton

Peebles P.J.E., 1993, Principles of the Physical Cosmology Princeton University Press, Princeton

Press W.H., and Schechter P. 1974, ApJ, 187, 425

Splinter R. J., Melott A.L., Shandarin S.F., and Suto, Y. 1998, ApJ, 497, 38

Syer D., and White S.D.M. 1998, MNRAS, 293, 337

Tormen G., Bouchet, F.R., and White S.D.M. 1997, MNRAS, 286, 865

Tyson J.A., Kochanski, G.P., and Dell'Antonio I.P. 1998, ApJ, 498, L107

Viana P.T.P., and Liddle A.R. 1996, MNRAS, 281, 323

White S.D.M., Navarro J.F., Evrard A.E., and Frenk C.S. 1993, Nature, 366, 429

Zhao, H. S. 1996, MNRAS, 278, 488

\section{APPENDIX A: AN ACCURATE ANALYTICAL FITTING FORMULA FOR THE DENSITY PROFILE OF A NONSINGULAR ISOTHERMAL SPHERE}

The isothermal sphere solution can be approximated quite accurately by the simple analytical function

$\tilde{\rho}=\frac{A}{a^{2}+\zeta^{2}}-\frac{B}{b^{2}+\zeta^{2}}$

as suggested by NL97. In what follows, we adopt an approach similar to that of NL97 to solve for the best values of the constants in equation (A1), different from those in NL97, so as to provide a better fit over the full range of radii required to describe the TIS solution derived in this paper. We note that, while the final result of NL97 in the body of their paper is correct, several formulae (specifically equations (A9) and (A11)) in their Appendix A2 are incorrect and thus the method should be exercised with care.

The solution of equation (29) has a Taylor expansion at $\zeta=0$ :

$\tilde{\rho}=1-\frac{1}{6} \zeta^{2}+\frac{1}{45} \zeta^{4}-\frac{61}{22680} \zeta^{6}+\frac{629}{2041200} \zeta^{8}+O\left(\zeta^{10}\right)$

Depending on our requirements on the solution, we can find different sets of parameters for the best fit. If, for example, we require that the first three terms of the Taylor series of equation A1 at $\zeta=0$ are the same as in equation A2 and that $\tilde{\rho}$ has the right behaviour at infinity,

$\tilde{\rho} \rightarrow 2 / \zeta^{2}$ as $\quad \zeta \rightarrow \infty$

then we obtain the simple approximation of equation (A2) with the following parameters

$\left(A, a^{2}, B, b^{2}\right)_{N L, 1}=(50,10,48,12)$.

This approximation is fairly accurate for small radii (fractional error less that 2 per cent for $\zeta<5$ ) and for very large radii (error $<2$ per cent for $\zeta>100$ ). However, in the intermediate interval, which we are most interested in, the fractional error of this approximation can be as large as 24 per cent. To remedy this problem, NL97 offers the following choice of parameters:

$\left(A, a^{2}, B, b^{2}\right)_{N L, 2}=(44.34,10.16,42.61,12.66)$.

(C) 0000 RAS, MNRAS 000, 000-000 


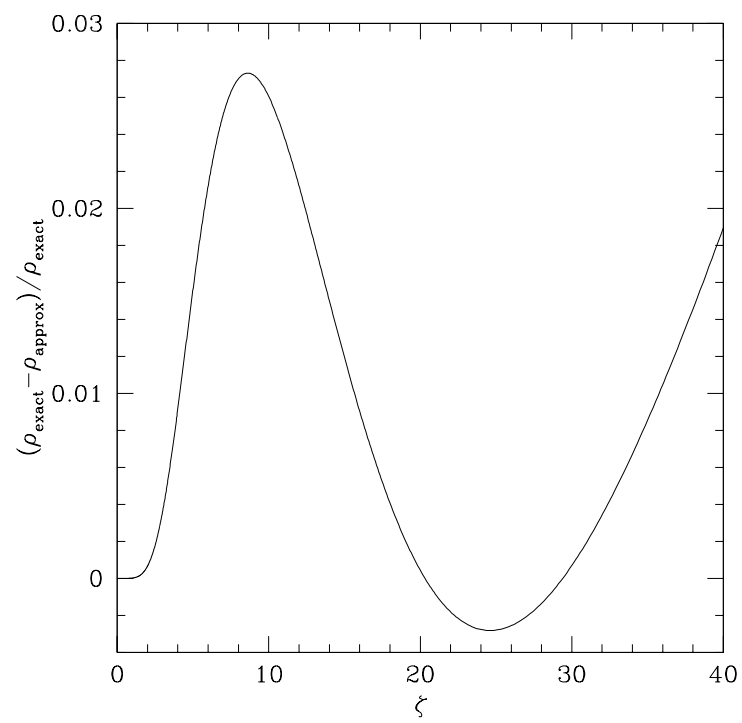

Figure A1. Fractional error $\left(\rho_{\text {exact }}-\rho_{\text {approx }}\right) / \rho_{\text {exact }}$ of the approximation of equations A1 and (A6).

This latter approximation is an extremely good fit up to $\zeta=10$, but deteriorates after that, having fractional errors of 5-8 per cent for $\zeta=15-40$. Hence, we have used their method, instead, to find an approximation which better suits our purposes, by requiring the first three terms of the Taylor series to match those in equation (A2), and by imposing an exact match at $\zeta=29.4$, which produces the result quoted in our equation (103) in the main text, namely

$\left(A, a^{2}, B, b^{2}\right)_{T I S}=(21.38,9.08,19.81,14.62)$.

While we cannot guarantee that this is the best possible approximation of this type, it is accurate to within 3 per cent for $\zeta<40$ (Fig A1), while for most of the interval the error is much smaller, which should be sufficient for many purposes where using a simple functional form is more important than obtaining a high precision. 